\title{
GEOMETRIC SOBOLEV-LIKE EMBEDDING USING HIGH-DIMENSIONAL MENGER-LIKE CURVATURE
}

\author{
SŁAWOMIR KOLASIŃSKI
}

Abstract. We study a modified version of Lerman-Whitehouse Menger-like curvature defined for $(m+2)$ points in an $n$-dimensional Euclidean space. For $1 \leq l \leq m+2$ and an $m$-dimensional set $\Sigma \subset R^{n}$, we also introduce global versions of this discrete curvature by taking the supremum with respect to $(m+2-l)$ points on $\Sigma$. We then define geometric curvature energies by integrating one of the global Menger-like curvatures, raised to a certain power $p$, over all $l$-tuples of points on $\Sigma$. Next, we prove that if $\Sigma$ is compact and $m$ Ahlfors regular and if $p$ is greater than the dimension of the set of all $l$-tuples of points on $\Sigma$ (i.e. $p>m l$ ), then the P. Jones' $\beta$-numbers of $\Sigma$ must decay as $r^{\tau}$ with $r \rightarrow 0$ for some $\tau \in(0,1)$. If $\Sigma$ is an immersed $C^{1}$ manifold or a bilipschitz image of such a set then, it follows that it is Reifenberg flat with vanishing constant; hence (by a theorem of David, Kenig and Toro) an embedded $C^{1, \tau}$ manifold. We also define a wide class of other sets for which this assertion is true. After that, we bootstrap the exponent $\tau$ to $\alpha=1-m l / p$, which is optimal due to our theorem with S. Blatt [Adv. Math., 2012]. This gives an analogue of the Morrey-Sobolev embedding theorem $W^{2, p}\left(\mathbb{R}^{m l}\right) \subseteq C^{1, \alpha}\left(\mathbb{R}^{m l}\right)$ but, more importantly, we also obtain a qualitative control over the local graph representations of $\Sigma$ only in terms of the energy.

\section{INTRODUCTION}

Menger curvature is defined for three points $x_{0}, x_{1}, x_{2}$ in $\mathbb{R}^{n}$ as follows:

$$
\mathbf{c}\left(x_{0}, x_{1}, x_{2}\right)=\frac{4 \mathcal{H}^{2}\left(\triangle\left(x_{0}, x_{1}, x_{2}\right)\right)}{\left|x_{0}-x_{1}\right|\left|x_{1}-x_{2}\right|\left|x_{2}-x_{0}\right|},
$$

where $\mathcal{H}^{l}$ denotes the $l$-dimensional Hausdorff measure and $\triangle\left(x_{0}, \ldots, x_{l}\right)$ is the convex hull of the set $\left\{x_{0}, \ldots, x_{l}\right\}$. Using the sine theorem one easily sees that $\mathbf{c}\left(x_{0}, x_{1}, x_{2}\right)$ is just the inverse of the radius of the circumcircle of $\triangle\left(x_{0}, x_{1}, x_{2}\right)$. Let $\gamma \subseteq \mathbb{R}^{3}$ be a closed, Lipschitz curve with arc-length parameterization $\Gamma$, i.e. $\Gamma: S_{L} \rightarrow \mathbb{R}^{3}$ is such that $\gamma=\Gamma\left(S_{L}\right)$ and $\left|\Gamma^{\prime}\right|=1$ a.e. (here $S_{L}=\mathbb{R} / L \mathbb{Z}$ denotes the circle of length $L)$. Set

$$
\begin{gathered}
\mathbf{c}_{0}[\gamma]=\sup _{x_{0}, x_{1}, x_{2} \in \gamma} \mathbf{c}\left(x_{0}, x_{1}, x_{2}\right), \quad \mathbf{c}_{1}[\gamma]\left(x_{0}\right)=\sup _{x_{1}, x_{2} \in \gamma} \mathbf{c}\left(x_{0}, x_{1}, x_{2}\right), \\
\mathbf{c}_{2}[\gamma]\left(x_{0}, x_{1}\right)=\sup _{x_{2} \in \gamma} \mathbf{c}\left(x_{0}, x_{1}, x_{2}\right) \quad \text { and } \quad \mathbf{c}_{3}[\gamma]\left(x_{0}, x_{1}, x_{2}\right)=\mathbf{c}\left(x_{0}, x_{1}, x_{2}\right) .
\end{gathered}
$$

Received by the editors May 24, 2012.

2010 Mathematics Subject Classification. Primary 49Q10; Secondary 28A75, 49Q20, 49Q15.

Key words and phrases. Menger curvature, Ahlfors regularity, repulsive potentials, regularity theory.

The major part of this work was accomplished while the author was working at the University of Warsaw and was supported by the Polish Ministry of Science grant no. N N201 611140. The work was put in its final form at the AEI Golm, AEI publication number AEI-2013-165. 
Using these quantities we define

$$
\neg[\gamma]=\mathbf{c}_{0}[\gamma]^{-1} \quad \text { and for } i=1,2,3 \quad \mathcal{M}_{p}^{i}(\gamma)=\int_{(\gamma)^{i}} \mathbf{c}_{i}^{p}[\gamma] d \mathcal{H}^{i},
$$

where $(\gamma)^{i}$ is the Cartesian product of $i$ copies of $\gamma$. Gonzalez and Maddocks 7 ] suggested that these functionals can serve as knot energies, i.e. energies which separate knot types by infinite energy barriers. Gonzalez, Maddocks, Schuricht and von der Mosel [6] showed that whenever $\mathbf{c}_{0}[\gamma]<\infty, \gamma$ is an embedded (without self-intersections) manifold of class $C^{1,1}=W^{2, \infty}$. The functionals $\mathcal{M}_{p}^{1}, \mathcal{M}_{p}^{2}$ and $\mathcal{M}_{p}^{3}$ possess a similar property. For $i=1,2,3$, if $\mathcal{M}_{p}^{i}(\gamma)<\infty$ for some $p>i$, then $\gamma$ is an embedded manifold of class $C^{1,1-i / p}$ (see the articles by Strzelecki, Szumańska and von der Mosel [23,24] and by Strzelecki and von der Mosel 25]). Furthermore, in 25] the authors proved that $\mathcal{M}_{p}^{1}(\gamma)$ is finite if and only if $\gamma$ is an image of a $W^{2, p}$ function. Later Blatt [2 showed that for $i=2,3$ and $p>i$ the energy $\mathcal{M}_{p}^{i}(\gamma)<\infty$ if and only if $\gamma$ belongs to the Sobolev-Slobodeckij space $W^{1+s, p}$, where $s=1-\frac{i-1}{p}$. Note that, $W^{1+s, p}(\mathbb{R}) \subseteq C^{1,1-i / p}(\mathbb{R})$ whenever $p>i$ and $1-i / p$ is the optimal Hölder exponent, so these results deliver geometric counterparts of the Sobolev-Morrey embedding.

For $p$ below the critical level (i.e. $p<i$ ) one cannot expect that finiteness of $\mathcal{M}_{p}^{i}(\gamma)$ implies smoothness. This can easily be seen by considering $\gamma=$ $\triangle((0,0),(1,0),(1,1))$-a triangle in the plane. Take a countable family of parallel lines $\left\{l_{k}\right\}_{k=1}^{\infty}$, such that $\operatorname{dist}\left(l_{k},(0,0)\right)=2^{-k}$. Observe that the energy $\mathcal{M}_{i}^{i}$ is invariant under scaling, so for each $k \in \mathbb{N}$ the part of $\gamma$ lying between the lines $l_{k}$ and $l_{k+1}$ has the same energy. Hence $\mathcal{M}_{i}^{i}(\gamma)=\infty$. For any $p<i$ we have $\mathcal{M}_{p}(\gamma)<\infty$ (see Scholtes [20, 21]).

The case $p=2$ and $l=3$ is particularly interesting. For a 1-dimensional Borel set $E \subseteq \mathbb{R}^{2}$ a famous result of David and Léger [15] says that $\mathcal{M}_{2}^{3}(E)$ is finite if and only if $E$ is rectifiable. This was a crucial step in the proof of Vitushkin's conjecture characterizing removable sets $E$ for bounded analytic functions.

There are some generalizations of these results to higher dimensions. Lerman and Whitehouse [16,17] suggested a few possible definitions of discrete curvatures of Menger-type. They used these curvatures to characterize uniformly rectifiable measures in the sense of David and Semmes [4. In this article we use a modified version (having different scaling) of one of the quantities introduced in [16].

Our research has been motivated directly by the work of Strzelecki and von der Mosel [26], where the authors work with 2-dimensional surfaces in $\mathbb{R}^{3}$. They define the discrete curvature of four points $x_{0}, x_{1}, x_{2}, x_{3} \in \mathbb{R}^{3}$ by the formula

$$
\mathcal{K}_{S v d M}\left(x_{0}, x_{1}, x_{2}, x_{3}\right)=\frac{\mathcal{H}^{3}\left(\triangle\left(x_{0}, x_{1}, x_{2}, x_{3}\right)\right)}{\mathcal{H}^{2}\left(\partial \triangle\left(x_{0}, x_{1}, x_{2}, x_{3}\right)\right) \operatorname{diam}\left(\triangle\left(x_{0}, x_{1}, x_{2}, x_{3}\right)\right)^{2}} .
$$

For $\Sigma \subseteq \mathbb{R}^{3}$ a closed, connected, Lipschitz surface they also define

$$
\mathcal{M}_{p}^{S v d M}(\Sigma)=\int_{\Sigma} \int_{\Sigma} \int_{\Sigma} \int_{\Sigma} \mathcal{K}_{S v d M}\left(x_{0}, x_{1}, x_{2}, x_{3}\right)^{p} d \mathcal{H}_{x_{0}}^{2} d \mathcal{H}_{x_{1}}^{2} d \mathcal{H}_{x_{2}}^{2} d \mathcal{H}_{x_{3}}^{2} .
$$

In [26] the authors prove that if $\mathcal{M}_{p}^{S v d M}(\Sigma) \leq E<\infty$ for some $p>8=\operatorname{dim}\left(\Sigma^{4}\right)$, then $\Sigma$ has to be an embedded manifold of class $C^{1,1-8 / p}$ with local graph representations whose domain size is controlled solely in terms of $E$ and $p$. This additional control of the graph representations allowed them to prove [26, Theorem 1.5] that 
any sequence $\left(\Sigma_{j}\right)_{j \in \mathbb{N}}$ of closed, connected, Lipschitz surfaces containing the origin and with uniformly bounded measure and energy, i.e. $\mathcal{M}_{p}^{S v d M}\left(\Sigma_{j}\right) \leq E$ and $\mathcal{H}^{2}\left(\Sigma_{j}\right) \leq A$ for each $j \in \mathbb{N}$, contains a subsequence $\Sigma_{j_{l}}$, which converges in $C^{1}$ topology to some $C^{1,1-8 / p}$ closed, connected manifold. This in turn allowed them to solve some variational problems with topological constraints (see [26, Theorems 1.6 and 1.7]).

Similar regularity results were also obtained by Strzelecki and von der Mosel [27] for yet another energy

$$
\mathcal{E}_{p}^{t p}(\Sigma)=\int_{\Sigma} \int_{\Sigma} R_{t p}(x, y)^{-p} d \mathcal{H}_{x}^{m} d \mathcal{H}_{y}^{m}, \quad \text { where } \quad R_{t p}(x, y)=\frac{|x-y|^{2}}{2 \operatorname{dist}\left(y-x, T_{x} \Sigma\right)}
$$

and $T_{x} \Sigma$ is the tangent space to $\Sigma$ at $x$. The quantity $R_{t p}(x, y)$ is called the tangentpoint radius, because it measures the radius of the sphere tangent to $\Sigma$ at $x$ and passing through $y$. If $\Sigma$ is a closed, connected, Lipschitz surface with $\mathcal{E}_{p}^{t p}(\Sigma)<\infty$ for some $p>2 m$, then $\Sigma \in C^{1,1-(2 m) / p}$.

In this paper we define energy functionals for $m$-dimensional subsets $\Sigma$ of $\mathbb{R}^{n}$ (we always assume $m \leq n$ ) and we study regularity of sets with finite energy. For $m+2$ points $x_{0}, \ldots, x_{m+1}$ in $\mathbb{R}^{n}$ we set (cf. [16, $\left.\S 6.1 .1\right]$ )

$$
\mathcal{K}\left(x_{0}, \ldots, x_{m+1}\right)=\frac{\mathcal{H}^{m+1}\left(\triangle\left(x_{0}, \ldots, x_{m+1}\right)\right)}{\operatorname{diam}\left(\triangle\left(x_{0}, \ldots, x_{m+1}\right)\right)^{m+2}}
$$

and for $p>0$ and $l=1,2, \ldots, m+2$ we define 1

$$
\mathcal{E}_{p}^{l}(\Sigma)=\int_{\Sigma^{l} x_{l}, \ldots x_{m+1} \in \Sigma} \sup _{\mathcal{K}} \mathcal{K}\left(x_{0}, \ldots, x_{m+1}\right)^{p} d \mathcal{H}_{x_{0}, \ldots, x_{l-1}}^{m l} .
$$

We prove that these functionals can be called geometric curvature energies, i.e. for sets $\Sigma$ of relatively little smoothness, finiteness of the energy guarantees both embeddedness and higher regularity.

Of course, the condition $\mathcal{E}_{p}^{l}(\Sigma)<\infty$ cannot guarantee that $\Sigma$ is a manifold (even for large $p$ ) just for any $m$-dimensional set $\Sigma$. The main issue is that $\mathcal{E}_{p}^{l}(\Sigma \backslash A) \leq$ $\mathcal{E}_{p}^{l}(\Sigma)$ for any set $A$, so creating holes in $\Sigma$ decreases the energy. Hence, we need to work with a restricted class of sets. We say that $\Sigma$ is locally lower Ahlfors regular if (Ahl) $\quad \exists R_{\mathrm{Ahl}}>0 \exists A_{\mathrm{Ahl}}>0 \forall x \in \Sigma \forall r \leq R_{\mathrm{Ahl}} \quad \mathcal{H}^{m}(\Sigma \cap \mathbb{B}(x, r)) \geq A_{\mathrm{Ahl}} r^{m}$.

Here $\mathbb{B}(x, r)$ denotes the $n$-dimensional open ball of radius $r$ centered at $x$. We also need a variant of the P. Jones' beta numbers introduced in [10] and the bilateral beta numbers, which originated from Reifenberg's work [19] and his famous topological disc theorem (see 22] for a modern proof). We define

$$
\begin{gathered}
\beta_{m}^{\Sigma}(x, r)=\frac{1}{r} \inf _{H \in G(n, m)} \sup _{z \in \Sigma \cap \overline{\mathbb{B}}(x, r)} \operatorname{dist}(z, x+H) \\
\text { and } \theta_{m}^{\Sigma}(x, r)=\frac{1}{r} \inf _{H \in G(n, m)} d_{\mathcal{H}}(\Sigma \cap \overline{\mathbb{B}}(x, r),(x+H) \cap \overline{\mathbb{B}}(x, r)), \\
\text { where } d_{\mathcal{H}}(E, F)=\sup _{y \in E} \operatorname{dist}(y, F)+\sup _{y \in F} \operatorname{dist}(y, E)
\end{gathered}
$$

is the Hausdorff distance and $G(n, m)$ denotes the Grassmannian of $m$-dimensional linear subspaces of $\mathbb{R}^{n}$. The $\beta$-number measures the flatness of $\Sigma$ in a given scale

\footnotetext{
${ }^{1}$ If $l=m+2$ there are $m+2$ integrals and no supremum.
} 
in a scaling invariant way. The $\theta$-number measures additionally the size of holes in that scale. Using these notions we can formulate our first

Proposition 1. Let $\Sigma \subseteq \mathbb{R}^{n}$ be a compact set satisfying Ahl and let $l \in\{1, \ldots$, $m+2\}$. If $\mathcal{E}_{p}^{l}(\Sigma) \leq E<\infty$ for some $p>m l$, then there exists a constant $C_{A}=$ $C_{A}(m, l, p)$ such that

$$
\forall r \leq R_{\text {Ahl }} \forall x \in \Sigma \quad \beta_{m}^{\Sigma}(x, r) \leq C_{A}\left(\frac{E}{A_{\mathrm{Ahl}}^{l}}\right)^{\frac{1}{\kappa}} r^{\frac{\lambda}{\kappa}},
$$

where $\kappa=(p+m l)(m+1)$ and $\lambda=p-m l$.

Applying the result of David, Kenig and Toro [3, Proposition 9.1] (cf. Proposition 1.3. we then obtain

Theorem 1. Let $\Sigma \subseteq \mathbb{R}^{n}$ be a compact set satisfying (AhI) and such that $(\theta \lesssim \beta) \quad \exists R_{\theta \beta}>0 \exists M_{\theta \beta}>1 \forall x \in \Sigma \forall r \leq R_{\theta \beta} \quad \theta_{m}^{\Sigma}(x, r) \leq M_{\theta \beta} \beta_{m}^{\Sigma}(x, r)$. If $\mathcal{E}_{p}^{l}(\Sigma)<\infty$ for some $p>m l$, then $\Sigma$ is a closed, embedded manifold of class $C^{1, \lambda / \kappa}$.

This motivates the following

Definition 1. We say that a set $\Sigma \subseteq \mathbb{R}^{n}$ is an $m$-fine set if it is $m$-dimensional, compact and satisfies (Ah] and $(\theta \lesssim \beta$.

Examples of $m$-fine sets include closed $m$-dimensional Lipschitz submanifolds of $\mathbb{R}^{n}$ and also images of maps $\varphi: M \rightarrow \mathbb{R}^{n}$, where $M$ is an abstract, closed $C^{1}$-manifold and $\varphi$ is an immersion. Other examples are described in Section 2.2 .

The condition $(\theta \lesssim \beta)$ is purely geometric but it is hard to understand what kind of behavior it implies. It gives control over the size of holes in $\Sigma$ but it does not imply that the topological boundary of $\Sigma$ is empty. In [27, Definition 2.9] (cf. Definition 3.2) the authors considered a class of admissible sets satisfying a different list of conditions. Their idea was to use the topological linking number to prevent appearance of holes in $\Sigma$. Any admissible set in the sense of [27] with finite $\mathcal{E}_{p^{-}}$. energy for some $p>m l$, satisfies the $(\theta \lesssim \beta)$ condition (see [13, Theorem 4.15] for the case $l=m+2$ ); hence, by Theorem 1, it is a closed $C^{1, \lambda / \kappa}$-manifold.

Once we have estimates on the $\beta$-numbers (Proposition 11), the regularity result (Theorem 1) follows quite easily but the key point is that one can get a uniform (not depending on $\Sigma$ ) control over the local graph representations of $\Sigma$ only in terms of the energy bound $E$ and the parameters $m, l$ and $p$. To show that this is true we first prove the following uniform, with respect to $\Sigma$, estimate on the local lower Ahlfors regularity of $\Sigma$.

Theorem 2. Let $\Sigma \subseteq \mathbb{R}^{n}$ be an $m$-fine set. If $\mathcal{E}_{p}^{l}(\Sigma) \leq E<\infty$ for some $p>m l$, then

$$
\exists R_{0}=R_{0}(E, m, l, p)>0 \forall x \in \Sigma \forall r \leq R_{0} \quad \mathcal{H}^{m}(\Sigma \cap \mathbb{B}(x, r)) \geq\left(\frac{\sqrt{15}}{4}\right)^{m} \omega_{m} r^{m},
$$

where $\omega_{m}=\mathcal{H}^{m}\left(\mathbb{B}(0,1) \cap \mathbb{R}^{m}\right)$ is the measure of the unit ball in $\mathbb{R}^{m}$.

Theorem 1 together with Theorem 2 give us estimates on the $\beta$-numbers independent of $\Sigma$. Knowing that $\Sigma$ is a closed, $C^{1, \lambda / \kappa}$-submanifold of $\mathbb{R}^{n}$, we also prove that the constant $M_{\theta \beta}$ from the $(\theta \lesssim \beta)$ condition can be replaced by an absolute constant. Then we obtain estimates on the oscillation of tangent planes of $\Sigma$ solely 
in terms of $E, m, l$ and $p$. This allows us to prove that the size of a single patch of $\Sigma$ representable as a graph of some function is controlled solely in terms of $E$, $m, l$ and $p$. Next we bootstrap the exponent $\frac{\lambda}{\kappa}$ to $\alpha=1-\frac{m l}{p}$.

Theorem 3. Let $\Sigma \subseteq \mathbb{R}^{n}$ be an $m$ fine set. If $\mathcal{E}_{p}^{l}(\Sigma) \leq E<\infty$ for some $p>m l$, then $\Sigma$ is a closed $C^{1, \alpha}$-manifold, where $\alpha=1-\frac{m l}{p}$. Moreover, there exist two positive constants $R_{\mathrm{g}}=C(m, l, p) E^{-1 / \lambda}$ and $C_{\mathrm{g}}=C_{\mathrm{g}}(E, m, l, p)$ such that

$$
\begin{gathered}
\forall x \in \Sigma \exists F_{x} \in C^{1, \alpha}\left(T_{x} \Sigma,\left(T_{x} \Sigma\right)^{\perp}\right) \quad \Sigma \cap \mathbb{B}\left(x, R_{\mathrm{g}}\right)=\operatorname{Graph}\left(F_{x}\right) \cap \mathbb{B}\left(x, R_{\mathrm{g}}\right) \\
\text { and } \quad \forall y, z \in T_{x} \Sigma \quad\left\|D F_{x}(y)-D F_{x}(z)\right\| \leq C_{\mathrm{g}}|y-z|^{\alpha},
\end{gathered}
$$

where $\operatorname{Graph}\left(F_{x}\right)=\left\{z \in \mathbb{R}^{n}: \exists y \in T_{x} \Sigma \quad z=y+F_{x}(y)\right\}$.

This work already led to a few other results. In our joint work with Szumańska [14] we have constructed an example of a function $f \in C^{1, \alpha_{0}}\left([0,1]^{m}\right)$, where $\alpha_{0}=$ $1-\frac{m(m+1)}{p}$, whose graph has infinite $\mathcal{E}_{p}^{m+2}$-energy and we proved that for any $\alpha_{1}>\alpha_{0}$ the graphs of $C^{1, \alpha_{1}}$ functions always have finite energy. Later this result was complemented by our joint work with Blatt [1, where we have shown that a closed $C^{1}$-submanifold of $\mathbb{R}^{n}$ has finite $\mathcal{E}_{p}^{l}$-energy for some $p>m(l-1)$ and $l \in\{2, \ldots, m+2\}$ if and only if it is locally a graph of a function in the SobolevSlobodeckij space $W^{1+s, p}$, where $s=1-\frac{m(l-1)}{p}$. Since $W^{1+s, p}\left(\mathbb{R}^{m}\right) \subseteq C^{1, \alpha}\left(\mathbb{R}^{m}\right)$, this also shows that the exponent $\alpha$ in Theorem 3 is optimal. In another article 12 written jointly with Strzelecki and von der Mosel, we have shown that an $m$-fine set $\Sigma \subseteq \mathbb{R}^{n}$ is a $W^{2, p}$-manifold if and only if it satisfies the condition $\mathcal{E}_{p}^{1}(\Sigma)<\infty$. The paper [12] includes Theorem 3 for the $\mathcal{E}_{p}^{1}$-energy and a counterpart of Theorem 3 for a modified version of the $\mathcal{E}_{p}^{t p}$-energy, where one integration was replaced by taking the supremum. In a forthcoming joint article with Strzelecki and von der Mosel [1] we also prove a compactness result similar to [26, Theorem 1.5] for the $\mathcal{E}_{p}^{l}$ and $\mathcal{E}_{p}^{t p}$ energies.

Organization of the paper. In Section 1 we describe the notation, we state precisely the result of [3] about Reifenberg flat sets with vanishing constant and we prove some auxiliary propositions about roughly regular simplices and about the metric on the Grassmannian. In Subsection 1.4 we also show that $C^{2}$-manifolds have finite $\mathcal{E}_{p}^{l}$-energy for any $p>0$. In Section 2 we prove Proposition 1 and Theorem 1 and we give some examples of $m$-fine sets. In Section 3 we establish Theorem 2. For this we need to define another class of admissible sets and prove some more auxiliary results about cones and homotopies inside cones. In Section 4 we prove a counterpart of Theorem 3 where $\alpha$ is replaced with $\lambda / \kappa$. In Section 5 we bootstrap the exponent $\lambda / \kappa$ to the optimal $\alpha=1-\frac{m l}{p}$ and consequently establish Theorem 3 .

\section{Preliminaries}

1.1. Notation. We write $\mathbb{S}$ for the unit $(n-1)$-dimensional sphere centered at the origin and we write $\mathbb{B}$ for the unit $n$-dimensional open ball centered at the origin. We also use the symbols $\mathbb{S}_{r}=r \mathbb{S}, \mathbb{B}_{r}=r \mathbb{B}, \mathbb{S}(x, r)=x+r \mathbb{S}$ and $\mathbb{B}(x, r)=x+r \mathbb{B}$.

If $v=\left(v_{1}, \ldots, v_{n}\right)$ is a vector in $\mathbb{R}^{n}$, we write $|v|=\sqrt{\sum\left|v_{i}\right|^{2}}=\sqrt{\langle v, v\rangle}$ for the standard Euclidean norm of $v$. If $\mathbf{A}: \mathbb{R}^{k} \rightarrow \mathbb{R}^{l}$ is a linear operator, we write $\|\mathbf{A}\|=\sup _{|v|=1}|\mathbf{A} v|$ for the operator norm of $\mathbf{A}$. 
The symbol $G(n, m)$ denotes the Grassmann manifold of $m$-dimensional linear subspaces of $\mathbb{R}^{n}$. Whenever we write $U \in G(n, m)$ we identify the point $U$ of the space $G(n, m)$ with the appropriate $m$-dimensional subspace of $\mathbb{R}^{n}$. In particular any vector $u \in U$ is treated as an $n$-dimensional vector in the ambient space $\mathbb{R}^{n}$ which happens to lie in $U \subseteq \mathbb{R}^{n}$.

If $A$ is any set, then we write $\operatorname{id}_{A}: A \rightarrow A$ for the identity mapping. Let $H \in G(n, m)$. We use the symbol $\pi_{H}$ to denote the orthogonal projection onto $H$ and $\pi_{H}^{\perp}=I-\pi_{H}$ to denote the orthogonal projection onto the orthogonal complement $H^{\perp}$. We write aff $\left\{x_{0}, \ldots, x_{m}\right\}$ for the smallest affine subspace of $\mathbb{R}^{n}$ containing the points $x_{0}, \ldots, x_{m} \in \mathbb{R}^{n}$, i.e.

$$
\operatorname{aff}\left\{x_{0}, \ldots, x_{m}\right\}=x_{0}+\operatorname{span}\left\{x_{1}-x_{0}, \ldots, x_{m}-x_{0}\right\} .
$$

Let $\mathbf{T}=\triangle\left(x_{0}, \ldots, x_{k}\right)$. We set

- $\mathfrak{f c}_{i} \mathbf{T}=\triangle\left(x_{0}, \ldots, \widehat{x}_{i}, \ldots, x_{k}\right)$ - the $i$-th face of $\mathbf{T}$,

- $\mathfrak{h}_{i}(\mathbf{T})=\operatorname{dist}\left(x_{i}, \operatorname{aff}\left\{x_{0}, \ldots, \widehat{x_{i}}, \ldots, x_{k}\right\}\right.$ - the height lowered from $x_{i}$,

- $\mathfrak{h}_{\min }(\mathbf{T})=\min \left\{\mathfrak{h}_{i}(\mathbf{T}): i=0,1, \ldots, k\right\}$ - the minimal height of $\mathbf{T}$.

In the course of the proofs we will frequently use cones and "conical caps" of different sorts. We define

- $\mathbb{C}(\delta, H)=\left\{x \in \mathbb{R}^{n}:\left|\pi_{H}^{\perp}(x)\right| \geq \delta|x|\right\}$ - the cone with "axis" $H^{\perp}$ and "angle" $\delta$,

- $\mathbb{A}(r, R)=\mathbb{B}_{R} \backslash \overline{\mathbb{B}}_{r}$ - the open shell (or the $n$-annulus) of radii $r$ and $R$,

- $\mathbb{C}(\delta, H, r, R)=\mathbb{C}(\delta, H) \cap \mathbb{A}(r, R)$ - the conical cap with "angle" $\delta$, "axis" $H^{\perp}$ and radii $r$ and $R$.

Remark. We use the notation $C=C(x, y, z)$ to denote that $C$ depends solely on $x$, $y$ and $z$. The symbols $C, \hat{C}, \tilde{C}, \bar{C}$ are used to denote general constants, whose values may change in different parts of the text. Subscripts in constants (like " $C_{\theta}$ ") do not denote dependences but are used to name the constant and distinguish it from other constants. Subscripted constants always have global meaning and do not change.

1.2. Reifenberg flat sets. For convenience we introduce the following

Definition 1.1. Let $\Sigma \subseteq \mathbb{R}^{n}$ be any set. Let $x \in \Sigma$ and $r>0$. We say that $H \in G(n, m)$ is the best approximating m-plane for $\Sigma$ in $\overline{\mathbb{B}}(x, r)$ and write $H \in$ $\operatorname{BAP}_{\mathrm{m}}(x, r)$ if the following condition is satisfied:

$$
d_{\mathcal{H}}(\Sigma \cap \overline{\mathbb{B}}(x, r),(x+H) \cap \overline{\mathbb{B}}(x, r)) \leq r \theta_{m}^{\Sigma}(x, r) .
$$

Since $G(n, m)$ is compact, such $H$ always exists, but it might not be unique, e.g. consider the set $\Sigma=\mathbb{S} \cup\{0\}$ and take $x=0, r=2$.

Recall the definitions of $\beta_{m}^{\Sigma}$ and $\theta_{m}^{\Sigma}$ given in the introduction. In [3], the authors define the $\beta$ and $\theta$ numbers in a slightly different way using open balls instead of closed ones. This does not change much since both definitions lead to comparable quantities (see [13, Proposition 1.35]).

Definition 1.2 (cf. [3], Definition 1.3). We say that a closed set $\Sigma \subseteq \mathbb{R}^{n}$ is Reifenberg-flat with vanishing constant (of dimension $m$ ) if for every compact subset $K \subseteq \Sigma$

$$
\lim _{r \rightarrow 0} \sup _{x \in K} \theta_{m}^{\Sigma}(x, r)=0 .
$$


The following proposition was proved by David, Kenig and Toro.

Proposition 1.3 (cf. [3], Proposition 9.1). Let $\tau \in(0,1)$ be given. Suppose $\Sigma$ is a Reifenberg-flat set with vanishing constant of dimension $m$ in $\mathbb{R}^{n}$ and that, for each compact subset $K \subseteq \Sigma$ there is a constant $C_{K}$ such that

$$
\beta_{m}^{\Sigma}(x, r) \leq C_{K} r^{\tau} \quad \text { for each } x \in K \text { and } r \leq 1 .
$$

Then $\Sigma$ is a $C^{1, \tau}$-submanifold of $\mathbb{R}^{n}$.

1.3. Voluminous simplices. Here we define the class of $(\eta, d)$-voluminous simplices, where $\eta$ measures the "regularity" of a simplex. The curvature $\mathcal{K}$ of any such simplex is controlled in terms of $\eta$ and $d$. A very similar notion was used by Lerman and Whitehouse in [16, §3.1], where these kinds of simplices were called 1-separated. We derive estimates of the distance by which we can move each vertex of an $(\eta, d)$ voluminous simplex without losing the lower bound on the curvature. We will use this result to obtain a lower bound on the $\mathcal{E}_{p}^{l}$-energy in the proof of Proposition 2.1.

Definition 1.4. Let $\mathbf{T}=\triangle\left(x_{0}, \ldots, x_{k}\right)$ be a simplex in $\mathbb{R}^{n}$ and let $d \in(0, \infty)$ and $\eta \in(0,1)$. We say that $\mathbf{T}$ is $(\eta, d)$-voluminous if

$$
\operatorname{diam}(\mathbf{T}) \leq d \quad \text { and } \quad \mathfrak{h}_{\min }(\mathbf{T}) \geq \eta d
$$

Remark 1.5. If $\mathbf{T}=\triangle T$ is $(\eta, d)$-voluminous, then

$$
\frac{(\eta d)^{k}}{k !} \leq \mathcal{H}^{k}(\mathbf{T}) \leq \frac{d^{k}}{k !} ; \quad \text { hence } \quad \mathcal{K}(T) \geq \frac{\eta^{k}}{k ! d} .
$$

Let us recall the definition of the outer product:

Definition 1.6. Let $w_{1}, \ldots, w_{l}$ be vectors in $\mathbb{R}^{n}$. We define the outer product

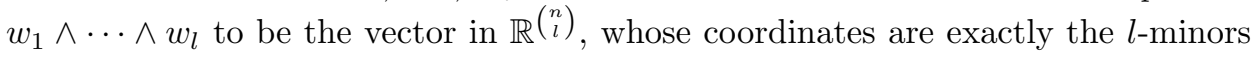
of the $(n \times l)$-matrix $\left(w_{1}, \ldots, w_{l}\right)$.

Remark. A standard fact from linear algebra says that the length $\left|w_{1} \wedge \cdots \wedge w_{l}\right|$ of the outer product of $w_{1}, \ldots, w_{l}$ is equal to the $l$-dimensional volume of the parallelotope spanned by $w_{1}, \ldots, w_{l}$. In particular $\left|w_{1} \wedge \cdots \wedge w_{l}\right| \leq\left|w_{1}\right| \cdot\left|w_{2}\right| \cdots\left|w_{k}\right|$.

Proposition 1.7. Let $\mathbf{T}_{0}=\triangle T_{0}=\triangle\left(x_{0}, \ldots, x_{k}\right)$ be an $(\eta, d)$-voluminous simplex in $\mathbb{R}^{n}$. There exists a number $\varsigma_{k}=\varsigma_{k}(\eta) \in(0,1)$ such that for any simplex $\mathbf{T}_{1}=$ $\triangle T_{1}=\triangle\left(y_{0}, \ldots, y_{k}\right)$ satisfying $\left|x_{i}-y_{i}\right| \leq \varsigma_{k}$ d for each $i=1, \ldots, k$ the estimate

$$
\frac{3}{4} \mathcal{H}^{k}\left(\mathbf{T}_{0}\right) \leq \mathcal{H}^{k}\left(\mathbf{T}_{1}\right) \leq \frac{5}{4} \mathcal{H}^{k}\left(\mathbf{T}_{0}\right) \quad \text { holds; hence also } \quad \mathcal{K}\left(T_{1}\right) \geq \frac{3 \eta^{k}}{2^{k+3} k ! d} .
$$

Proof. Let $\tilde{\varsigma} \in(0,1)$ be some number and let $T_{1}=\left(y_{0}, \ldots, y_{k}\right)$ be such that $\left|x_{i}-y_{i}\right| \leq \tilde{\varsigma} d$ for each $i=1, \ldots, k$. We set $v_{i}=x_{i}-x_{0}$ and $w_{i}=\left(y_{i}-y_{0}\right)-v_{i}$, where $i=1, \ldots, k$.

$$
\begin{aligned}
\mathcal{H}^{k}\left(\mathbf{T}_{1}\right)= & \frac{1}{k !}\left|\left(v_{1}+w_{1}\right) \wedge \ldots \wedge\left(v_{k}+w_{k}\right)\right| \\
= & \frac{1}{k !} \mid\left(v_{1} \wedge \ldots \wedge v_{k}\right)+\left(w_{1} \wedge v_{2} \wedge \ldots \wedge v_{k}\right)+\left(v_{1} \wedge w_{2} \wedge \ldots \wedge v_{k}\right) \\
& \quad+\ldots+\left(w_{1} \wedge w_{2} \wedge v_{3} \wedge \ldots \wedge v_{k}\right)+\ldots+\left(w_{1} \wedge w_{2} \wedge w_{3} \wedge \ldots \wedge w_{k}\right) \mid .
\end{aligned}
$$


Whenever we take an outer product of $j$ vectors from the set $\left\{w_{1}, \ldots, w_{k}\right\}$ and $(k-j)$ vectors from the set $\left\{v_{1}, \ldots, v_{k}\right\}$ we obtain a vector of length at most $d^{k-j}(2 \tilde{\varsigma} d)^{j}$. Hence we can write

$\left|\left(w_{1} \wedge v_{2} \wedge \ldots \wedge v_{k}\right)+\ldots+\left(w_{1} \wedge w_{2} \wedge \ldots \wedge w_{k}\right)\right| \leq \sum_{j=1}^{k}\left(\begin{array}{c}k \\ j\end{array}\right) d^{k} 2^{j} \tilde{\varsigma}^{j}=d^{k}\left((1+2 \tilde{\varsigma})^{k}-1\right)$,

which gives $\mathcal{H}^{k}\left(\mathbf{T}_{0}\right)-d^{k}\left((1+2 \tilde{\varsigma})^{k}-1\right) \leq \mathcal{H}^{k}\left(\mathbf{T}_{1}\right) \leq \mathcal{H}^{k}\left(\mathbf{T}_{0}\right)+d^{k}\left((1+2 \tilde{\varsigma})^{k}-1\right)$. Since $\mathbf{T}_{0}$ is $(\eta, d)$-voluminous, it satisfies $\mathcal{H}^{k}\left(\mathbf{T}_{0}\right) \geq \frac{1}{k !}(\eta d)^{k}$. We set

$$
\varsigma_{k}=\frac{1}{2}\left(1+\frac{\eta^{k}}{4 k !}\right)^{\frac{1}{k}}-\frac{1}{2}
$$

so that $d^{k}\left((1+2 \tilde{\varsigma})^{k}-1\right) \leq \frac{1}{4} \mathcal{H}^{k}\left(\mathbf{T}_{0}\right)$. Thus, if $\left|x_{i}-y_{i}\right| \leq \varsigma_{k} d$, then we obtain the desired estimate $\frac{3}{4} \mathcal{H}^{k}\left(\mathbf{T}_{0}\right) \leq \mathcal{H}^{k}\left(\mathbf{T}_{1}\right) \leq \frac{5}{4} \mathcal{H}^{k}\left(\mathbf{T}_{0}\right)$.

Remark 1.8. Let $x, s \in \mathbb{R}$ and $s>0$. When $|x| \approx 0$, the function $(1+x)^{s}$ behaves asymptotically like $1+s x$; hence there exists a constant $C_{\varsigma}=C_{\varsigma}(k)>1$ such that

$$
\forall \eta \in(0,1) \quad \frac{1}{C_{\varsigma}} \eta^{k} \leq \varsigma_{k}(\eta) \leq C_{\varsigma} \eta^{k}
$$

1.4. The $\mathcal{E}_{p}^{l}$-energy for smooth manifolds. Observe that $\mathcal{K}(\alpha T)=\frac{1}{\alpha} \mathcal{K}(T)$ for any $\alpha>0$, so our curvature behaves under scaling like the original Menger curvature c. If $\triangle T$ is a regular simplex (meaning that all the side lengths are equal), then $\mathcal{K}(T) \simeq \frac{1}{\operatorname{diam} T} \simeq R(T)^{-1}$, where $R(T)$ is the radius of a circumsphere of $T$. For $m=1$ one easily sees that we always have $\mathcal{K}(T) \leq c(T)=R^{-1}(T)$. In dimension $m=2$ we also have $\mathcal{K}(T) \leq 4 \pi \mathcal{K}_{S v d M}(T)$ for any $T$ and $\mathcal{K}(T) \simeq \mathcal{K}_{S v d M}(T)$ if $T$ is a regular simplex.

We emphasize the behavior on regular simplices because small, close to regular (or voluminous) simplices are the reason why $\mathcal{E}_{p}^{l}(\Sigma)$ might get very big or infinite. For the class of $(\eta, d)$-voluminous simplices $T$ the value $\mathcal{K}(T)$ is comparable with yet another possible definition of discrete curvature (cf. [17, §10])

$$
\mathcal{K}^{\prime}(T)=\frac{\mathfrak{h}_{\min }(\triangle T)}{\operatorname{diam}(T)^{2}}=\frac{1}{\operatorname{diam}(T)} \frac{\mathfrak{h}_{\min }(\triangle T)}{\operatorname{diam}(T)},
$$

which is basically $\frac{1}{\operatorname{diam}(T)}$ multiplied by a scale-invariant "regularity coefficient" $\frac{\mathfrak{h}_{\min }(\triangle T)}{\operatorname{diam}(T)}$. This last factor prevents $\mathcal{K}^{\prime}$ from blowing up on simplices with vertices on smooth manifolds.

It occurs that one cannot define $k$-dimensional Menger curvature using integrals of $R^{-1}$. This "obvious" generalization of the Menger curvature fails because of examples (see [26, Appendix B]) of smooth embedded manifolds for which this kind of curvature would be unbounded. For the curvature $\mathcal{K}$ we have the following.

Proposition 1.9. If $M \subseteq \mathbb{R}^{n}$ is a compact, $m$-dimensional, $C^{2}$-manifold embedded in $\mathbb{R}^{n}$, then the discrete curvature $\mathcal{K}$ is bounded on $M^{m+2}$. Therefore $\mathcal{E}_{p}^{l}(M)$ is finite for every $p>0$ and every $l \in\{1, \ldots, m+2\}$.

Lemma 1.10. Let $\Sigma \subseteq \mathbb{R}^{n}$ be any set and let $T=\left(x_{0}, \ldots, x_{m+1}\right) \in \Sigma^{m+2}$. We set $\mathbf{T}=\triangle T$ and $d=\operatorname{diam}(\mathbf{T})$. There exists a constant $C_{\mathcal{K} \beta}=C_{\mathcal{K} \beta}(m, n)$ such that we have

$$
\mathcal{H}^{m+1}(\mathbf{T}) \leq C_{\mathcal{K} \beta} \beta_{m}^{\Sigma}\left(x_{0}, d\right) d^{m+1} \quad \text { and consequently } \quad \mathcal{K}(T) \leq C_{\mathcal{K} \beta} \frac{\beta_{m}^{\Sigma}\left(x_{0}, d\right)}{d}
$$


Proof.2 Without loss of generality we can assume that $x_{0}=0$. If the vectors $\left\{x_{1}, \ldots, x_{m+1}\right\}$ are not linearly independent, then $\mathcal{H}^{m+1}(\mathbf{T})=0$ and there is nothing to prove.

Let $x_{1}, \ldots, x_{m+1}$ be linearly independent and let $W$ denote the $(m+1)$ dimensional vector space spanned be these vectors. Set

$$
\mathbf{S}=\left\{s \in W^{\perp}:|s| \leq \beta_{m}^{\Sigma}\left(x_{0}, d\right) d\right\} .
$$

Then, the set $\mathbf{T}+\mathbf{S}$ is isometric with $\mathbf{T} \times \mathbf{S}$ and the following holds:

$$
\mathcal{H}^{n}(\mathbf{T}+\mathbf{S})=\mathcal{H}^{m+1}(\mathbf{T}) \mathcal{H}^{n-m-1}(\mathbf{S})=\omega_{n-m-1} \mathcal{H}^{m+1}(\mathbf{T}) d^{n-m-1} \beta_{m}^{\Sigma}(0, d)^{n-m-1} .
$$

Using compactness of the Grassmannian we can find a vector space $V \in G(n, m)$ such that

$$
\sup _{y \in \Sigma \cap \overline{\mathbb{B}}\left(x_{0}, d\right)}\left|\pi_{V}^{\perp}(y)\right|=\beta_{m}^{\Sigma}\left(x_{0}, d\right) d .
$$

The vertices of $\mathbf{T}$ lie in $\Sigma \cap \overline{\mathbb{B}}\left(x_{0}, d\right)$ and $\mathbf{T}$ is convex, so we also have

$$
\forall t \in \mathbf{T} \quad\left|\pi_{V}^{\perp}(t)\right| \leq \beta_{m}^{\Sigma}\left(x_{0}, d\right) d .
$$

Let $y \in \mathbf{T}+\mathbf{S}$ and let $t \in \mathbf{T}$ and $s \in \mathbf{S}$ be such that $s+t=y$. Using the triangle inequality we see that

$$
\begin{gathered}
\left|\pi_{V}(y)\right| \leq|y| \leq\left(1+\beta_{m}^{\Sigma}(0, d)\right) d \\
\text { and } \quad\left|\pi_{V}^{\perp}(y)\right| \leq\left|\pi_{V}^{\perp}(t)\right|+\left|\pi_{V}^{\perp}(s)\right| \leq 2 \beta_{m}^{\Sigma}\left(x_{0}, d\right) d .
\end{gathered}
$$

Hence, $\mathbf{T}+\mathbf{S}$ is a subset of

$$
Z=\left\{y \in \mathbb{R}^{n}:\left|\pi_{V}(y)\right| \leq 2 d,\left|\pi_{V}^{\perp}(y)\right| \leq 2 \beta_{m}^{\Sigma}(0, d) d\right\}
$$

and we obtain

$$
\mathcal{H}^{n}(\mathbf{T}+\mathbf{S}) \leq \mathcal{H}^{n}(Z)=\omega_{m} \omega_{n-m} 2^{n} \beta_{m}^{\Sigma}(0, d)^{n-m} d^{n} .
$$

Combining (4) and (5) we obtain the desired estimate.

Corollary 1.11. Let $\Sigma \subseteq \mathbb{R}^{n}$ be any set and let $T=\left(x_{0}, \ldots, x_{m+1}\right) \in \Sigma^{m+2}$. There exists a constant $C_{\eta \beta}=C_{\eta \beta}(n, m)$ such that if $\triangle T$ is $(\eta, d)$-voluminous, then the parameters $\eta$ and $d$ must satisfy

$$
\eta \leq C_{\eta \beta} \beta_{m}^{\Sigma}\left(x_{0}, d\right)^{\frac{1}{m+1}} .
$$

Proof. Recalling Remark [1.5 we have the estimate

$$
\mathcal{H}^{m+1}(\triangle T) \geq((m+1) !)^{-1}(\eta d)^{m+1},
$$

which, combined with Lemma 1.10, leads to $\eta \leq\left((m+1) ! C_{\mathcal{K} \beta}\right)^{\frac{1}{m+1}} \beta_{m}^{\Sigma}\left(x_{0}, d\right)^{\frac{1}{m+1}}$.

Proof of Proposition 1.9. Since $M$ is a compact $C^{2}$-manifold, it has a tubular neighborhood

$$
M_{\varepsilon}=M+\bar{B}_{\varepsilon}=\left\{x+y: x \in M, y \in \bar{B}_{\varepsilon}\right\}
$$

of some radius $\varepsilon>0$ and the nearest point projection $p: M_{\varepsilon} \rightarrow M$ is a well-defined, continuous function (see e.g. [5]).

\footnotetext{
${ }^{2}$ The author wishes to thank S. Blatt for significantly simplifying this proof while we were working on [1].
} 
We will show that for all $r \leq \varepsilon$ and all $x \in M$ we have

$$
\beta_{m}^{M}(x, r) \leq \frac{1}{2 \varepsilon} r .
$$

Next, we apply Lemma 1.10 and get the desired result.

Choose $r \in(0, \varepsilon]$. Fix some point $x \in M$ and pick a point $y \in T_{x} M^{\perp}$ with $|x-y|=\varepsilon$. Note that $y$ belongs to the tubular neighborhood $M_{\varepsilon}$ and applying [5, Theorem 4.8] we see that $p(y)=x$. Hence, the point $x$ is the only point of $M$ in the ball $\overline{\mathbb{B}}(y, \varepsilon)$. In other words $M \backslash\{x\}$ lies in the complement of $\overline{\mathbb{B}}(y, \varepsilon)$. This is true for any $y$ satisfying $y \in T_{x} M^{\perp}$ and $|x-y|=\varepsilon$, so we have

$$
M \subseteq \mathbb{R}^{n} \backslash \bigcup\left\{\overline{\mathbb{B}}(y, \varepsilon): y \perp T_{x} M,|y-x|=\varepsilon\right\} .
$$

Pick another point $\bar{x} \in M \cap \overline{\mathbb{B}}(x, r)$. We then have

$$
\bar{x} \in \overline{\mathbb{B}}(x, r) \backslash \bigcup\left\{\overline{\mathbb{B}}(y, \varepsilon): y \perp T_{x} M,|y-x|=\varepsilon\right\} .
$$

Using (7) and simple trigonometry, it is easy to calculate the maximal distance of $\bar{x}$ from the tangent space $T_{x} M$. Let $z$ be any point in the intersection $\partial \mathbb{B}(x, r) \cap$ $\partial \mathbb{B}(y, \varepsilon)$. Note that points of $M \cap \overline{\mathbb{B}}(x, \varepsilon)$ must be closer to $T_{x} M$ than $z$. In other words

$$
\forall x \in M \cap \mathbb{B}(x, r) \quad \operatorname{dist}\left(x, T_{x} M\right) \leq \operatorname{dist}\left(z, T_{x} M\right) .
$$

This situation is presented in Figure 1 Let $\alpha$ be the angle between $T_{x} M$ and $z$ and set $h=\operatorname{dist}\left(z, T_{x} M\right)$. We use the fact that the distance $|z-x|$ is equal to $r$.

$$
\sin \alpha=\frac{|z-x|}{2 \varepsilon}=\frac{h}{|z-x|} \Rightarrow h=\frac{|z-x|^{2}}{2 \varepsilon}=\frac{r^{2}}{2 \varepsilon} .
$$

This shows (6) and thus finishes the proof.

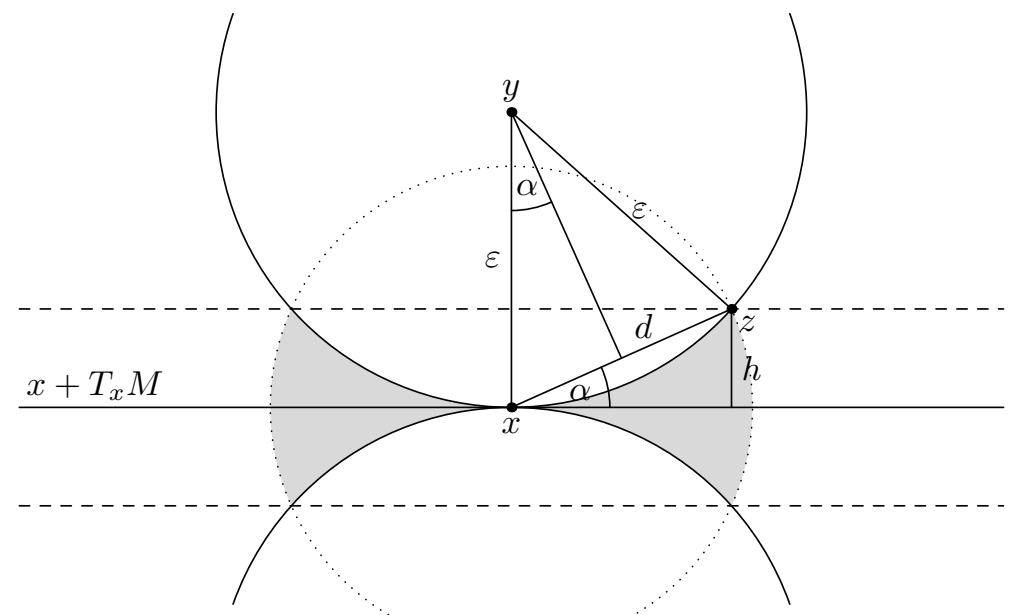

Figure 1. All of $M \cap \overline{\mathbb{B}}(x, r)$ lies in the grey area. The point $\bar{x}$ lies in the complement of $\mathbb{B}(y, \varepsilon)$ and inside $\overline{\mathbb{B}}(x, r)$ so it can only be as far from $T_{x} M$ as $z$ is. 
Remark 1.12. Note that the only property of $M$, which allowed us to prove Proposition [1.9, was the existence of an appropriate tubular neighborhood $M_{\varepsilon}$. One can easily see that Proposition 1.9 still holds if $M$ is a compact submanifold of positive reach as defined in [5].

1.5. The metric on the Grassmannian. Recall that, formally, $G(n, m)$ is defined as the homogeneous space

$$
G(n, m)=O(n) /(O(m) \times O(n-m)),
$$

where $O(n)$ is the orthogonal group; see e.g. Hatcher's book [8, §4.2, Examples $4.53,4.54$ and 4.55$]$ for the reference. We treat $G(n, m)$ as a metric space with the following metric.

Definition 1.13. Let $U, V \in G(n, m)$. We define the metric

$$
d_{\mathrm{Gr}}(U, V)=\left\|\pi_{U}-\pi_{V}\right\|=\sup _{w \in \mathbb{S}}\left|\pi_{U}(w)-\pi_{V}(w)\right| .
$$

Note that this metric is different from the geodesic distance on the Grassmannian. However, the topology induced by the metric $d_{\mathrm{Gr}}$ agrees with the standard quotient topology which is the same as the topology induced by the geodesic distance.

Remark 1.14. Let $I: \mathbb{R}^{n} \rightarrow \mathbb{R}^{n}$ denote the identity mapping. We will frequently use the following identity without reference:

$$
d_{\mathrm{Gr}}(U, V)=\left\|\pi_{U}-\pi_{V}\right\|=\left\|I-\pi_{U}^{\perp}-\left(I-\pi_{V}^{\perp}\right)\right\|=\left\|\pi_{V}^{\perp}-\pi_{U}^{\perp}\right\| .
$$

Definition 1.15. Let $V \in G(n, m)$ and let $\left(v_{1}, \ldots, v_{m}\right)$ be the basis of $V$. Fix some radius $\rho>0$ and a small constant $\varepsilon \in(0,1)$. We say that $\left(v_{1}, \ldots, v_{m}\right)$ is a $\rho \varepsilon$-basis if

$$
\forall i, j \in\{1, \ldots, m\} \quad\left(\delta_{i}^{j}-\varepsilon\right) \rho^{2} \leq\left|\left\langle v_{i}, v_{j}\right\rangle\right| \leq\left(\delta_{i}^{j}+\varepsilon\right) \rho^{2} .
$$

Here $\delta_{i}^{j}$ denotes the Kronecker delta.

Proposition 1.16. Let $\left(v_{1}, \ldots, v_{m}\right)$ be a $\rho \varepsilon$-basis of $V \in G(n, m)$ with constants $\rho=\rho_{0}>0$ and $\varepsilon=\varepsilon_{0} \in(0,1)$. Let $\left(u_{1}, \ldots, u_{m}\right)$ be some basis of $U \in G(n, m)$, such that $\left|u_{i}-v_{i}\right| \leq \vartheta \rho_{0}$ for some $\vartheta>0$ and for each $i=1, \ldots, m$. There exist constants $C_{\rho \varepsilon}=C_{\rho \varepsilon}(m)$ and $\boldsymbol{\epsilon}_{\rho \varepsilon}=\boldsymbol{\epsilon}_{\rho \varepsilon}(m)$ such that whenever $\varepsilon_{0} \leq \boldsymbol{\epsilon}_{\rho \varepsilon}$, then

$$
d_{\mathrm{Gr}}(U, V) \leq C_{\rho \varepsilon} \vartheta \text {. }
$$

Lemma 1.17. Let $\left(v_{1}, \ldots, v_{m}\right)$ be a $\rho \varepsilon$-basis of $V \in G(n, m)$ with constants $\rho=$ $\rho_{0}=1$ and $\varepsilon=\varepsilon_{0} \in(0,1)$. There exists an orthonormal basis $\hat{v}_{1}, \ldots, \hat{v}_{m}$ of $V$ and a constant $C_{g s}=C_{g s}(m)$ such that $\left|v_{i}-\hat{v}_{i}\right| \leq C_{g s} \varepsilon_{0}$.

Proof. Set

$$
\hat{v}_{1}=\frac{v_{1}}{\left|v_{1}\right|}, \quad s_{i}=\sum_{j=1}^{i-1}\left\langle v_{i}, \hat{v}_{j}\right\rangle \hat{v}_{j}, \quad \tilde{v}_{i}=v_{i}-s_{i} \quad \text { and } \quad \hat{v}_{i}=\frac{\tilde{v}_{i}}{\left|\tilde{v}_{i}\right|} .
$$

We proceed by induction. For $i=1$, we have $\left|\hat{v}_{1}-v_{1}\right|=|1-| v_{1}|| \leq \varepsilon_{0}$. Assume that for $i=1, \ldots, i_{0}-1$ we have $\left|\hat{v}_{i}-v_{i}\right|=C \varepsilon_{0}$ for some constant $C=C(i)$. It follows that

$$
\left|v_{i_{0}}-\tilde{v}_{i_{0}}\right|=\left|s_{i_{0}}\right| \leq \sum_{j=1}^{i_{0}-1}\left|\left\langle v_{i_{0}}, v_{j}\right\rangle\right|+\left|\left\langle v_{i_{0}}, \hat{v}_{j}-v_{j}\right\rangle\right| \leq \hat{C}\left(i_{0}\right) \varepsilon_{0}
$$


and

$$
1-\left(\hat{C}\left(i_{0}\right)+1\right) \varepsilon_{0} \leq\left|v_{i_{0}}\right|-\left|s_{i_{0}}\right| \leq\left|\tilde{v}_{i_{0}}\right| \leq\left|v_{i_{0}}\right|+\left|s_{i_{0}}\right| \leq 1+\left(\hat{C}\left(i_{0}\right)+1\right) \varepsilon_{0} ;
$$

hence $\left|v_{i_{0}}-\hat{v}_{i_{0}}\right| \leq\left|v_{i_{0}}-\tilde{v}_{i_{0}}\right|+\left|\tilde{v}_{i_{0}}-\hat{v}_{i_{0}}\right| \leq\left(2 \hat{C}\left(i_{0}\right)+1\right) \varepsilon_{0}$.

Lemma 1.18. Let $\left(\hat{v}_{1}, \ldots, \hat{v}_{m}\right)$ be an orthonormal basis of $V \in G(n, m)$ and let $U \in G(n, m)$ be such that $\left|\pi_{U}^{\frac{1}{U}}\left(\hat{v}_{i}\right)\right| \leq \vartheta$. There exists a constant $C_{\pi}=C_{\pi}(m)$ such that $d_{\mathrm{Gr}}(U, V) \leq C_{\pi} \vartheta$.

Proof. Without loss of generality, we can assume that $\vartheta<1$. If $\vartheta \geq 1$, then we can set $C_{\pi}=2$ and there is nothing to prove. Set $u_{i}=\pi_{U} \hat{v}_{i}$. Since $u_{i}=\hat{v}_{i}-\pi_{U} \frac{1}{\hat{v}_{i}}$ and $\left\langle\hat{v}_{i}, \hat{v}_{j}\right\rangle=\delta_{i}^{j}$, we have

$$
\delta_{i}^{j}-\left(\vartheta^{2}+2 \vartheta\right) \leq\left|\left\langle u_{i}, u_{j}\right\rangle\right| \leq \delta_{i}^{j}+\left(\vartheta^{2}+2 \vartheta\right),
$$

so $u_{1}, \ldots, u_{m}$ is a $\rho \varepsilon$-basis with $\rho=1$ and $\varepsilon=3 \vartheta$. From Lemma 1.17 there exists an orthonormal basis $\hat{u}_{1}, \ldots, \hat{u}_{m}$ such that $\left|u_{i}-\hat{u}_{i}\right| \leq 3 C_{g s} \vartheta$. Hence $\left|\hat{v}_{i}-\hat{u}_{i}\right| \leq$ $3 C_{g s} \vartheta+\vartheta \leq\left(1+3 C_{g s}\right) \vartheta$.

We calculate

$$
\begin{aligned}
d_{\mathrm{Gr}}(U, V) & =\sup _{w \in \mathbb{S}}\left|\pi_{U}(w)-\pi_{V}(w)\right|=\sup _{w \in \mathbb{S}}\left|\sum_{i=1}^{m}\left\langle w, \hat{u}_{i}\right\rangle \hat{u}_{i}-\left\langle w, \hat{v}_{i}\right\rangle \hat{v}_{i}\right| \\
& \leq \sup _{w \in \mathbb{S}} \sum_{i=1}^{m}\left|\left\langle w, \hat{u}_{i}\right\rangle\left(\hat{u}_{i}-\hat{v}_{i}\right)\right|+\left|\left\langle w,\left(\hat{u}_{i}-\hat{v}_{i}\right)\right\rangle \hat{v}_{i}\right| \leq 2 m\left(1+3 C_{g s}\right) \vartheta
\end{aligned}
$$

Proof of Proposition 1.16. Dividing each $v_{i}$ by $\rho_{0}$, we get a $\rho \varepsilon$-basis with $\rho=1$. Hence we can assume that $\rho_{0}=1$. Without loss of generality we may also assume that $\vartheta<1$. Indeed, we always have the trivial estimate $d_{\mathrm{Gr}}(U, V) \leq 2$, so if $\vartheta \geq 1$ we can set $C_{\rho \varepsilon}=2$.

Let $\hat{v}_{1}, \ldots, \hat{v}_{m}$ be the orthonormal basis given by Lemma 1.17applied to $v_{1}, \ldots$, $v_{m}$. Then

$\left|\pi_{U}^{\perp} \hat{v}_{i}\right| \leq\left|\pi_{U}^{\perp}\left(\hat{v}_{i}-v_{i}\right)\right|+\left|\pi_{U}^{\perp} v_{i}\right| \leq\left|\hat{v}_{i}-v_{i}\right| d_{\mathrm{Gr}}(U, V)+\left|v_{i}-u_{i}\right| \leq C_{g s} \varepsilon_{0} d_{\mathrm{Gr}}(U, V)+\vartheta$ for each $i=1, \ldots, m$. We set $\boldsymbol{\epsilon}_{\rho \varepsilon}=\boldsymbol{\epsilon}_{\rho \varepsilon}(m)=\frac{1}{2}\left(C_{\pi} C_{g s}\right)^{-1}$ and we assume $\varepsilon_{0} \leq \boldsymbol{\epsilon}_{\rho \varepsilon}$. Applying Lemma 1.18 we obtain the estimate

$$
d_{\mathrm{Gr}}(U, V) \leq C_{\pi} C_{g s} \varepsilon_{0} d_{\mathrm{Gr}}(U, V)+C_{\pi} \vartheta \quad \Longleftrightarrow \quad d_{\mathrm{Gr}}(U, V) \leq \frac{C_{\pi}}{1-C_{\pi} C_{g s} \varepsilon_{0}} \vartheta .
$$

\section{Geometric Morrey-Sobolev embedding}

In this section we prove Theorem 1 which is a geometric counterpart of the Morrey-Sobolev embedding $W^{2, p}\left(\mathbb{R}^{k}\right) \subseteq C^{1,1-k / p}$ for $p>k$. We also give some examples of $m$-fine sets to which Theorem 1 applies.

\subsection{Proof of Theorem 1.}

Proposition 2.1. Let $l \in\{1,2, \ldots, m+2\}$ and $p>m l$. Assume $\Sigma \subseteq \mathbb{R}^{n}$ satisfies (AhI) and also $\mathcal{E}_{p}^{l}(\Sigma) \leq E<\infty$. Let $T_{0}=\left(x_{0}, \ldots, x_{m+1}\right) \in \Sigma^{m+2}$. If $\mathbf{T}_{0}=\triangle T_{0}$ is $(\eta, d)$-voluminous with $d \leq R_{\mathrm{Ahl}}$, then $\eta$ and $d$ must satisfy

$$
d \geq\left(\frac{C_{\eta d} A_{\mathrm{Ahl}}^{l}}{E}\right)^{1 / \lambda} \eta^{\kappa / \lambda} \quad \text { or equivalently } \eta \leq\left(\frac{E}{C_{\eta d} A_{\mathrm{Ahl}}^{l}}\right)^{1 / \kappa} d^{\lambda / \kappa},
$$

where $C_{\eta d}=C_{\eta d}(m, l, p)$ is some constant, $\lambda=p-m l$ and $\kappa=(p+m l)(m+1)$. 
Proof. We shall estimate the $\mathcal{E}_{p}^{l}$-energy of $\Sigma$. Recall that $\varsigma_{m+1} \leq \frac{1}{4}$ was defined by (2).

$$
\begin{aligned}
& \text { (12) } \infty>E \geq \mathcal{E}_{p}^{l}(\Sigma)=\int_{\Sigma^{l} y_{l}, \ldots, y_{m+1} \in \Sigma} \sup \mathcal{K}^{p}\left(y_{0}, \ldots, y_{m+1}\right) d \mathcal{H}_{\left(y_{0}, \ldots, y_{l-1}\right)}^{m l} \\
& \geq \int_{\Sigma \cap \mathbb{B}\left(x_{0}, \varsigma_{m+1} d\right)} \cdots \int_{\Sigma \cap \mathbb{B}\left(x_{l-1}, \varsigma_{m+1} d\right)} \sup _{y_{l}, \ldots, y_{m+1} \in \Sigma} \mathcal{K}^{p}\left(y_{0}, \ldots, y_{m+1}\right) d \mathcal{H}_{\left(y_{0}, \ldots, y_{l}-1\right)}^{m l} .
\end{aligned}
$$

Proposition 1.7 combined with Remark 1.5 lets us estimate the integrand

$$
\sup _{y_{l}, \ldots, y_{m+1} \in \Sigma} \mathcal{K}^{p}\left(y_{0}, \ldots, y_{m+1}\right) \geq\left(\frac{3 \eta^{m+1}}{2^{m+4}(m+1) ! d}\right)^{p} .
$$

Since $\Sigma$ satisfies (Ahl), we get a lower bound on the measure of the sets over which we integrate

$$
\mathcal{H}^{m}\left(\Sigma \cap \mathbb{B}\left(x_{i}, \varsigma_{m+1} d\right)\right) \geq A_{\mathrm{Ahl}}\left(\varsigma_{m+1} d\right)^{m} .
$$

Plugging the last two estimates into (12) and recalling (3) we obtain

$$
E \geq\left(A_{\mathrm{Ahl}}\left(\varsigma_{m+1} d\right)^{m}\right)^{l}\left(\frac{3 \eta^{m+1}}{2^{m+4}(m+1) ! d}\right)^{p}=C_{\eta d}(m, l, p) A_{\mathrm{Ahl}}^{l} d^{m l-p} \eta^{(p+m l)(m+1)} .
$$

Proposition 2.1 is interesting in itself. It says that whenever the energy of $\Sigma$ is finite, we cannot have very small and voluminous simplices with vertices on $\Sigma$. It gives a bound on the "regularity" (i.e. parameter $\eta$ ) of any simplex in terms of its diameter $d$ and we see that $\eta$ goes to 0 when we decrease $d$. Now we are ready to prove Proposition 1 .

Proof of Proposition 1, Fix some point $x \in \Sigma$ and a radius $r \in\left(0, R_{\mathrm{Ahl}}\right)$. Let $\mathbf{T}=\triangle T=\triangle\left(x_{0}, \ldots, x_{m+1}\right)$ be an $(m+1)$-simplex such that $x_{i} \in \Sigma \cap \overline{\mathbb{B}}(x, r)$ for $i=0,1, \ldots, m+1$ and such that $\mathbf{T}$ has maximal $\mathcal{H}^{m+1}$-measure among all simplices with vertices in $\Sigma \cap \overline{\mathbb{B}}(x, r)$.

$$
\mathcal{H}^{m+1}(\mathbf{T})=\max \left\{\mathcal{H}^{m+1}\left(\triangle\left(x_{0}^{\prime}, \ldots, x_{m+1}^{\prime}\right)\right): x_{i}^{\prime} \in \Sigma \cap \bar{B}(x, r)\right\} .
$$

The existence of such simplex follows from the fact that the set $\Sigma \cap \overline{\mathbb{B}}(x, r)$ is compact and from the fact that the function $T \mapsto \mathcal{H}^{m+1}(\triangle T)$ is continuous with respect to $x_{0}, \ldots, x_{m+1}$.

Rearranging the vertices of $\mathbf{T}$ we can assume that $\mathfrak{h}_{\min }(\mathbf{T})=\mathfrak{h}_{m+1}(\mathbf{T})$, so the largest $m$-face of $\mathbf{T}$ is $\triangle\left(x_{0}, \ldots, x_{m}\right)$. Let $H=\operatorname{span}\left\{x_{1}-x_{0}, \ldots, x_{m}-x_{0}\right\}$, so that $x_{0}+H$ contains the largest $m$-face of $\mathbf{T}$. Note that the distance of any point $y \in \Sigma \cap \overline{\mathbb{B}}(x, r)$ from the affine plane $x_{0}+H$ has to be less than or equal to $\mathfrak{h}_{\text {min }}(\mathbf{T})=\operatorname{dist}\left(x_{m+1}, x_{0}+H\right)$. If we could find a point $y \in \Sigma \cap \overline{\mathbb{B}}(x, r)$ with $\operatorname{dist}\left(y, x_{0}+H\right)>\mathfrak{h}_{\min }(\mathbf{T})$, then the simplex $\triangle\left(x_{0}, \ldots, x_{m}, y\right)$ would have larger $\mathcal{H}^{m+1}$-measure than $\mathbf{T}$ but this is impossible due to the choice of $\mathbf{T}$.

Since $x \in \Sigma \cap \overline{\mathbb{B}}(x, r)$, we know that $\operatorname{dist}\left(x, x_{0}+H\right) \leq \mathfrak{h}_{\min }(\mathbf{T})$, so we obtain

$$
\forall y \in \Sigma \cap \overline{\mathbb{B}}(x, r) \quad \operatorname{dist}(y, x+H) \leq 2 \mathfrak{h}_{\min }(\mathbf{T}) .
$$

Now we only need to estimate $\mathfrak{h}_{\min }(\mathbf{T})=\mathfrak{h}_{m+1}(\mathbf{T})$ from above. Of course $\mathbf{T}$ is $\left(\mathfrak{h}_{\min }(\mathbf{T}) /(2 r), 2 r\right)$-voluminous, so applying Proposition 2.1] we obtain

$$
\frac{\mathfrak{h}_{\min }(\mathbf{T})}{2 r} \leq\left(\frac{E}{C_{\eta d} A_{\mathrm{Ahl}}^{l}}\right)^{1 / \kappa}(2 r)^{\lambda / \kappa} .
$$


Putting (13) and (14) together we get

$$
\beta_{m}^{\Sigma}(x, r) \leq \frac{2 \mathfrak{h}_{\min }(\mathbf{T})}{r} \leq 4\left(\frac{E}{C_{\eta d} A_{\mathrm{Ahl}}^{l}}\right)^{1 / \kappa}(2 r)^{\lambda / \kappa}=C(m, l, p)\left(\frac{E}{A_{\mathrm{Ahl}}^{l}}\right)^{1 / \kappa} r^{\lambda / \kappa} .
$$

Having Proposition 1 at our disposal, we can easily prove Theorem 1]

Proof of Theorem 1. We know already that $\beta_{m}^{\Sigma}(x, r) \leq C\left(m, l, p, A_{\mathrm{Ahl}}, E\right) r^{\lambda / \kappa}$ for $r<R_{\mathrm{Ahl}}$. We assumed $(\theta \lesssim \beta)$, so $\Sigma$ is Reifenberg-flat with vanishing constant. We finish the proof by applying Proposition 1.3 .

2.2. Examples of $m$-fine sets. Here we give a few examples of $m$-fine sets.

Example 2.2. Let $M$ be any $m$-dimensional, compact, closed manifold of class $C^{1}$ and let $f: M \rightarrow \mathbb{R}^{n}$ be an immersion. Then the image $\Sigma=\operatorname{im}(f)$ is an $m$ fine set. At each point $x \in M$, there is a radius $R_{x}$ such that a neighborhood $U_{x} \subseteq f^{-1}\left(\mathbb{B}\left(f(x), R_{x}\right)\right)$ of $x$ in $M$ is mapped to the set $V_{x}=f\left(U_{x}\right) \subseteq \mathbb{B}\left(f(x), R_{x}\right)$ and is a graph of some Lipschitz function $\Phi_{x}: D f(x) T_{x} M \rightarrow\left(D f(x) T_{x} M\right)^{\perp}$. If we choose $R_{x}$ small, then we can make the Lipschitz constant of $\Phi_{x}$ smaller than some $\varepsilon>0$. Due to the compactness of $M$ and continuity of $D f$ we can find a global radius $R_{\Sigma}=\min \left\{R_{x}: x \in M\right\}$. Then conditions (AhI) and ( $\left.\theta \lesssim \beta\right)$ are satisfied with $A_{\mathrm{Ahl}}=\sqrt{1-\varepsilon^{2}}, R_{\mathrm{Ahl}}=R_{\theta \beta}=R_{\Sigma}$ and $M_{\theta \beta}=4$.

Example 2.3. Let $\Sigma$ be the van Koch snowflake in $\mathbb{R}^{2}$. Then $\Sigma$ is 1 -fine but it fails to be 1-dimensional.

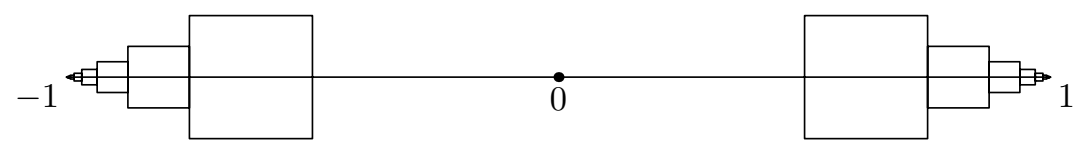

Figure 2. This set is 1-fine despite the fact that it has boundary points.

Example 2.4. Let $m=1, n=2$ and

$$
\Sigma=\bigcup_{k=1}^{\infty}\left(-Q_{k}\right) \cup\left\{(t, 0) \in \mathbb{R}^{2}: t \in[-1,1]\right\} \cup \bigcup_{k=1}^{\infty} Q_{k},
$$

$$
\text { where } Q_{0}=\partial([0,1] \times[0,1]) \quad \text { and } \quad Q_{k}=\left(\sum_{j=1}^{k} 2^{-j},-\frac{1}{2}\right)+2^{-(k+1)} Q_{0} .
$$

See Figure 2 for a graphical presentation. Condition $(\theta \lesssim \beta$ holds at the boundary points $(-1,0)$ and $(1,0)$ of $\Sigma$, because the $\beta$-numbers do not converge to zero with $r \rightarrow 0$ at these points. All the other points of $\Sigma$ are internal points of line segments or corner points of squares, so at these points condition $\theta \lesssim \beta$ is also satisfied. Hence, $\Sigma$ is 1-fine.

This example shows that condition $(\theta \lesssim \beta)$ does not exclude boundary points, but at any such boundary point we have to add some oscillation to prevent $\beta$ numbers from getting too small. The same effect can be observed in the following example:

$$
\Sigma=\partial([1,2] \times[-1,1]) \cup \overline{\left\{\left(x, x \sin \left(\frac{1}{x}\right)\right): x \in(0,1]\right\}} .
$$




\section{Uniform Ahlfors Regularity-the proof of Theorem 2}

Here we give the proof of Theorem 2, First we introduce the class of admissible sets, which is tailored for proving the existence of many voluminous simplices (cf. Proposition 3.18) with vertices on $\Sigma$. Proposition 3.18 is crucial in the proof of Theorem 2. In the end we also show how to make all the emerging constants depend solely on $E, m, l$ and $p$.

3.1. The class of admissible sets. In this section we introduce the definition of the class $\mathcal{A}(\delta, m)$ of $(\delta, m)$-admissible sets-here $\delta \in(0,1)$ is some number. This definition is essentially the same as [27, Definition 2.9] but it is more convenient for us to impose only local lower Ahlfors regularity (Ahl) instead of Condition H1 of [27. Definition 2.9].

Definition 3.1. Let $I$ be a countable set of indices and assume there exist compact, closed, $m$-dimensional manifolds $M_{i}$ of class $C^{1}$, a set $Z$ with $\mathcal{H}^{m}(Z)=0$ and continuous maps $f_{i}: M_{i} \rightarrow \mathbb{R}^{n}$ for $i \in I$, such that

$$
\Sigma=\bigcup_{i \in I} f_{i}\left(M_{i}\right) \cup Z \text {. }
$$

Let $N$ be an $(n-m)$-dimensional, compact, closed submanifold of $\mathbb{R}^{n}$ such that $f_{i}\left(M_{i}\right) \cap N=\emptyset$ for all $i \in I$. We say that $\Sigma$ is linked with $N$ and write $\operatorname{lk}_{2}(\Sigma, N)=1$, if there exists an $i \in I$ such that the map

$$
F: M_{i} \times N \rightarrow \mathbb{S}^{n-1}, \quad F(w, z)=\frac{f_{i}(w)-z}{\left|f_{i}(w)-z\right|} \text { satisfies } \operatorname{deg}_{2} F=1,
$$

where $\mathrm{deg}_{2}$ is the topological degree modulo 2.

For the definition of the degree of a map we refer the reader to [9, Chapter 5, $\S 1]$.

Definition 3.2 (cf. 27] Definition 2.9). Let $\delta \in(0,1)$ and let $I$ be a countable set of indices. Let $\Sigma$ be a compact subset of $\mathbb{R}^{n}$ satisfying (Ah]). We say that $\Sigma$ is $(\delta, m)$-admissible and write $\Sigma \in \mathcal{A}(\delta, m)$ if the following conditions are satisfied:

A1 Mock tangent planes and flatness. There exists a dense subset $\Sigma^{*} \subseteq \Sigma$ of full measure in $\Sigma$ (i.e. $\mathcal{H}^{m}\left(\Sigma \backslash \Sigma^{*}\right)=0$ ) such that for each $x \in \Sigma^{*}$ there is an $m$-plane $H=H_{x} \in G(n, m)$ and a radius $r_{0}=r_{0}(x)>0$ such that

$$
\left|\pi_{H}^{\perp}(y-x)\right|<\delta|y-x| \text { for each } y \in \mathbb{B}\left(x, r_{0}\right) \cap \Sigma .
$$

A2 Structure and linking. There exist compact, closed, $m$-dimensional manifolds $M_{i}$ of class $C^{1}$, a set $Z$ with $\mathcal{H}^{m}(Z)=0$ and continuous maps $f_{i}: M_{i} \rightarrow \mathbb{R}^{n}$ for $i \in I$, such that

$$
\Sigma=\bigcup_{i \in I} f_{i}\left(M_{i}\right) \cup Z
$$

$$
\text { and } \forall x \in \Sigma^{*} \quad \operatorname{lk}_{2}\left(\Sigma, \mathscr{S}_{x}\right)=1 \text { where } \mathscr{S}_{x}=\mathbb{S}\left(x, \frac{1}{2} r_{0}\right) \cap\left(x+H_{x}^{\perp}\right) .
$$

Condition A1 ensures that at every point $x \in \Sigma^{*}$ one can touch $\Sigma$ with an appropriate cone. Condition A2 says that at each point of $\Sigma$ there is a sphere $\mathscr{S}_{x}$ which is linked with $\Sigma$. This means intuitively that we cannot move $\mathscr{S}_{x}$ far away from $\Sigma$ without tearing one of these sets. Example 3.10 shows that this condition is unavoidable for the theorems stated in this paper to be true.

There are three especially useful properties of $\mathrm{lk}_{2}$ that we want to use. 
Proposition 3.3 (cf. 27, Lemma 3.2). Let $A \subseteq \mathbb{R}^{n}$ be a $(\delta, m)$-admissible set and let $N$ be a compact, closed $(n-m-1)$-dimensional manifold of class $C^{1}$, and let $N_{j}=h_{j}(N)$ for $j=0,1$, where $h_{j}$ is a $C^{1}$-embedding of $N$ into $\mathbb{R}^{n}$ such that $N_{j} \cap \Sigma=\emptyset$. If there is a homotopy

$$
G: N \times[0,1] \rightarrow \mathbb{R}^{n} \backslash \Sigma,
$$

such that $G(-, 0)=h_{0}$ and $G(-, 1)=h_{1}$, then

$$
\operatorname{lk}_{2}\left(\Sigma, N_{0}\right)=\mathrm{lk}_{2}\left(\Sigma, N_{1}\right) .
$$

Proposition 3.4 (cf. [27, Lemma 3.4). Let $\Sigma \subseteq \mathbb{R}^{n}$ be a $(\delta, m)$-admissible set. Choose $y \in \mathbb{R}^{n}$ and $\varepsilon \in \mathbb{R}$ such that $0<\varepsilon<r<2 \varepsilon$ and $\operatorname{dist}(y, \Sigma) \geq 3 \varepsilon$. Then

$$
\mathrm{lk}_{2}(\Sigma, \mathbb{S}(y, r) \cap(y+V))=0
$$

for each $V \in G(n, n-m)$.

Proposition 3.5 (cf. [27, Lemma 3.5). Let $\Sigma \subseteq \mathbb{R}^{n}$ be a $(\delta, m)$-admissible set. Assume that for some $y \in \mathbb{R}^{n}, r>0$ and $V \in G(n, n-m)$ we have

$$
\mathrm{lk}_{2}(\Sigma, \mathbb{S}(y, r) \cap(y+V))=1 .
$$

Then the disk $\mathbb{B}(y, r) \cap(y+V)$ contains at least one point of $\Sigma$.

Example 3.6. Let $\Sigma$ be any closed, compact, $m$-dimensional submanifold of $\mathbb{R}^{n}$ of class $C^{1}$. Then $\Sigma \in \mathcal{A}(\delta, m)$ for any $\delta \in(0,1)$.

It is easy to verify that $\Sigma \in \mathcal{A}(\delta, m)$. Take $M_{1}=\Sigma$ and $f_{1}=\operatorname{id}_{M_{1}}$. The set $Z$ will be empty, so $\Sigma^{*}=\Sigma$. At each point $x \in \Sigma$ we set $H_{x}$ to be the tangent space $T_{x} \Sigma$. Small spheres centered at $x \in \Sigma$ and contained in $x+H_{x}^{\perp}$ are linked with $\Sigma$; for the proof see e.g. [18, pp. 194-195]. Note that we do not assume orientability; that is why we used degree modulo 2 .

Example 3.7. Let $\Sigma=\bigcup_{i=1}^{N} \Sigma_{i}$, where $\Sigma_{i}$ are closed, compact, $m$-dimensional submanifolds of $\mathbb{R}^{n}$ of class $C^{1}$. Moreover assume that these manifolds intersect only on sets of zero $m$-dimensional Hausdorff measure, i.e.

$$
\mathcal{H}^{m}\left(\Sigma_{i} \cap \Sigma_{j}\right)=0 \text { for } i \neq j .
$$

Then $\Sigma \in \mathcal{A}(\delta, m)$ for any $\delta \in(0,1)$.

Remark 3.8. Any compact $C^{1}$-manifold is $(\delta, m)$-admissible (cf. Example 3.6) for any $\delta \in(0,1)$; hence any $m$-fine set with finite $\mathcal{E}_{p}^{l}$-energy for some $p>m l$ is also $(\delta, m)$-admissible.

It turns out that any $(\delta, m)$-admissible set with finite $\mathcal{E}_{p}^{l}$-energy for some $p>m l$ is also $m$-fine. We will not use this fact in this article. The proof for the $\mathcal{E}_{p}^{m+2}$ energy can be found in [13, Theorem 2.13].

If we do not assume finiteness of the $\mathcal{E}_{p}^{l}$-energy, then these two classes of sets are different and none of them is contained in the other.

Example 3.9. Let

$$
\Sigma=([0,1] \times\{0\}) \cup(\{1\} \times[0,1]) \cup\left(\left\{\left(x, x^{2}\right): x \in[0,1]\right\}\right) \subseteq \mathbb{R}^{2} .
$$

Then $\Sigma$ is $(\delta, 1)$-admissible for any $\delta \in(0,1)$ but it is not 1-fine. It does not satisfy $(\theta \lesssim \beta)$ at the points $(0,0)$ and $(1,1)$.

Now we give some negative examples showing the role of condition $\mathrm{A2}$. 
Example 3.10. Let $H \in G(n, m)$ and let $\Sigma=\pi_{H}(\mathbb{S})=\overline{\mathbb{B}} \cap H$. Then $\Sigma$ satisfies (AhI) and condition A1 but it does not satisfy A2. Hence, it is not admissible. Although $\Sigma$ is a compact, $m$-dimensional submanifold of $\mathbb{R}^{n}$ of class $C^{1}$, it is not closed.

Example 3.11. Let $\Sigma=\mathbb{S} \cap \mathbb{R}^{m+1}$. Of course $\Sigma$ is admissible as it falls into the case presented in Example 3.6. We want to emphasize that there are good and bad decompositions of $\Sigma$ into the sum $\bigcup f_{i}\left(M_{i}\right)$ from condition A2.

The easiest one and the best one is to set $M_{1}=\Sigma$ and $f_{1}=\mathrm{id}_{M_{1}}$. But there are other possibilities. Set $M_{1}=\mathbb{S} \cap \mathbb{R}^{m+1}$ and $M_{2}=\mathbb{S} \cap \mathbb{R}^{m+1}$ and set

$$
\begin{aligned}
& f_{1}\left(x_{1}, \ldots, x_{m+1}\right)=\left(x_{1}, \ldots, x_{m},\left|x_{m+1}\right|\right), \\
& f_{2}\left(x_{1}, \ldots, x_{m+1}\right)=\left(x_{1}, \ldots, x_{m},-\left|x_{m+1}\right|\right),
\end{aligned}
$$

so that $f_{1}$ maps $M_{1}$ to the upper hemisphere and $f_{2}$ maps $M_{2}$ to the lower hemisphere. This decomposition is bad, because (15) is not satisfied at any point.

3.2. Homotopies inside cones. In this section we prove a few useful facts about cones. In the proof of Proposition 3.18 we construct a set $F$ by glueing conical caps together. Then we need to know that we can deform one sphere lying in $F$ to some other sphere lying in $F$ without leaving $F$. To be able to do this easily we need Propositions 3.16 and 3.17

Definition 3.12. Let $H \in G(n, m)$ be an $m$-dimensional subspace of $\mathbb{R}^{n}$ and let $\delta \in(0,1)$ be some number. We define the set

$$
\mathscr{G}(\delta, H)=\left\{V \in G(n, n-m): \forall v \in V\left|\pi_{H}^{\perp}(v)\right| \geq \delta|v|\right\} .
$$

In other words $V \in \mathscr{G}(\delta, H)$ if and only if $V$ is contained in the cone $C(\delta, H)$. If $n=3$ and $m=1$, then $H$ is a line in $\mathbb{R}^{3}$ and the cone $C(\delta, H)$ contains all the 2-dimensional planes $V$ such that $\sin (\varangle(H, V)) \geq \delta$.

Proposition 3.13 (cf. [13, Proposition 4.2). For any two spaces $U$ and $V$ in $\mathscr{G}(\delta, H)$ there exists a continuous path $\gamma:[0,1] \rightarrow \mathscr{G}(\delta, H)$ such that $\gamma(0)=V$ and $\gamma(1)=U$.

Corollary 3.14 (cf. [13, Corollary 4.3). The path $\gamma$ from Proposition 3.13 lifts to a continuous path $\tilde{\gamma}:[0,1] \rightarrow O(n)$ in the orthogonal group.

The proof of Proposition 3.13 is quite elementary and Corollary 3.14 follows from the homotopy lifting property of fiber bundles (see [8, Proposition 4.48]). The details of the proofs can be found in [13, Section 4.1.1].

Corollary 3.15. Let $H$ and $\delta$ be as in Proposition 3.13. Let $S_{1}$ and $S_{2}$ be two round spheres centered at the origin, contained in the conical cap $\mathbb{C}\left(\delta, H, \rho_{1}, \rho_{2}\right)$ and of the same dimension $(n-m-1)$. Moreover assume that $0 \leq \rho_{1}<\rho_{2}$. There exists an isotopy

$$
\begin{gathered}
F: S_{1} \times[0,1] \rightarrow \mathbb{C}\left(\delta, H, \rho_{1}, \rho_{2}\right), \\
\text { such that } \quad F(-, 0)=\operatorname{id}_{S_{1}} \quad \text { and } \quad F\left(S_{1} \times\{1\}\right)=S_{2} .
\end{gathered}
$$

Proof. Let $r_{1}$ and $r_{2}$ be the radii of $S_{1}$ and $S_{2}$ respectively. We have $\rho_{1}<r_{1}, r_{2}<$ $\rho_{2}$. Let $V_{1}, V_{2} \in G(n, n-m)$ be the two subspaces of $\mathbb{R}^{n}$ such that $S_{1} \subseteq V_{1}$ and $S_{2} \subseteq V_{2}$. In other words $S_{1}=\mathbb{S}_{r_{1}} \cap V_{1}$ and $S_{2}=\mathbb{S}_{r_{2}} \cap V_{2}$. Because $S_{1}$ and $S_{2}$ are subsets of $\mathbb{C}(\delta, H)$, we know that $V_{1}$ and $V_{2}$ are elements of $\mathscr{G}(\delta, H)$. From Proposition 3.13 we get a continuous path $\gamma$ joining $V_{1}$ with $V_{2}$. By Corollary 3.14 . 
this path lifts to a path $\tilde{\gamma}$ in the orthogonal group $O(n)$. For $z \in S_{1}$ and $t \in[0,1]$ we set

$$
F(z, t)=\tilde{\gamma}(t) \tilde{\gamma}(0)^{-1} z .
$$

This gives a continuous deformation of $S_{1}=\mathbb{S}_{r_{1}} \cap V_{1}$ into $\mathbb{S}_{r_{1}} \cap V_{2}$. Now, we only need to adjust the radius but this can be easily done inside $V_{2} \cap \mathbb{A}\left(\rho_{1}, \rho_{2}\right)$ so the corollary is proven.

Proposition 3.16. Let $H \in G(n, m)$. Let $S$ be a sphere perpendicular to $H$, meaning that $S=\mathbb{S}(x, r) \cap\left(x+H^{\perp}\right)$ for some $x \in H$ and $r>0$. Assume that $S$ is contained in the conical cap $\mathbb{C}\left(\delta, H, \rho_{1}, \rho_{2}\right)$, where $\rho_{2}>0$. Fix some $\rho \in\left(\rho_{1}, \rho_{2}\right)$. There exists an isotopy

$$
F: S \times[0,1] \rightarrow \mathbb{C}\left(\delta, H, \rho_{1}, \rho_{2}\right),
$$

such that $F(\cdot, 0)=\operatorname{id}_{S} \quad$ and $\quad F(S \times\{1\})=\mathbb{S}_{\rho} \cap H^{\perp}$.

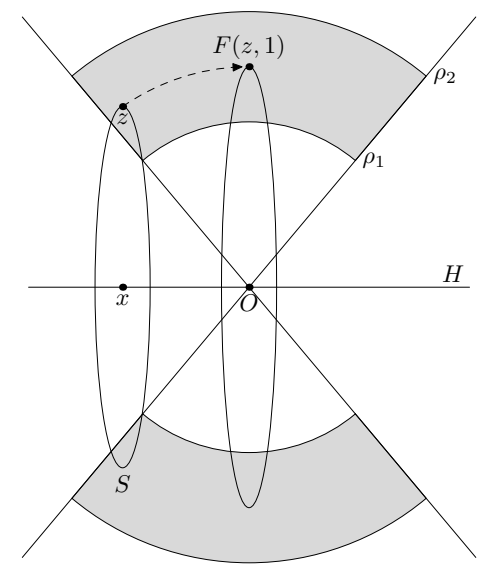

FiguRE 3 . When we move the center of a sphere to the origin, we need to control the radius so that the deformation is performed inside the conical cap.

Proof. Any point $z \in S$ can be uniquely decomposed into a sum $z=x+r y$, where $y \in \mathbb{S} \cap H^{\perp}$ is a point in the unit sphere in $H^{\perp}$. We define

$$
F(x+r y, t)=(1-t) x+y \sqrt{r^{2}+|x|^{2}-|(1-t) x|^{2}} .
$$

This gives an isotopy which deforms $S$ to a sphere perpendicular to $H$ and centered at the origin (see Figure 3). Fix some $z=x+r y \in S$. The sphere $S$ is contained in $\mathbb{C}(\delta, H)$, so it follows that

$$
\frac{\left|\pi_{H}^{\perp}(F(z, t))\right|}{|F(z, t)|}=\frac{\sqrt{r^{2}+|x|^{2}-|(1-t) x|^{2}}}{\sqrt{r^{2}+|x|^{2}}} \geq \frac{r}{\sqrt{r^{2}+|x|^{2}}}=\frac{\left|\pi_{H}^{\perp}(z)\right|}{|z|} \geq \delta .
$$

This shows that the whole deformation is performed inside $\mathbb{C}(\delta, H)$. Next, we need to continuously change the radius to the value $\rho$ but this can be easily done inside $H^{\perp} \cap\left(\mathbb{B}_{\rho_{2}} \backslash \mathbb{B}_{\rho_{1}}\right)$. 
Next, we give a sufficient condition on $\alpha$ and $\beta$ assuring that $\mathbb{C}(\alpha, P) \cap \mathbb{C}(\beta, H)$ contains another cone $\mathbb{C}(\gamma, H)$ for some $\gamma \in(0,1)$. This allows us to construct homotopies of spheres inside $\mathbb{C}(\alpha, P) \cup \mathbb{C}(\beta, H)$.

Proposition 3.17. Let $\alpha>0$ and $\beta>0$ be two real numbers satisfying $\alpha+\beta<$ $\sqrt{1-\beta^{2}}$ and let $H_{0}, H_{1} \in G(n, m)$ be two m-planes in $\mathbb{R}^{n}$. Assume that

$$
\mathbb{C}\left(\sqrt{1-\alpha^{2}}, H_{0}^{\perp}\right) \cap \mathbb{C}\left(\sqrt{1-\beta^{2}}, H_{1}^{\perp}\right) \neq \emptyset .
$$

Then for any $\epsilon>0$ we have the inclusion

$$
\mathbb{C}\left((\alpha+\beta) / \sqrt{1-\beta^{2}}+\epsilon, H_{0}\right) \subseteq \mathbb{C}\left(\epsilon, H_{1}\right) .
$$

In particular, if $\alpha+\beta \leq(1-\beta) \sqrt{1-\beta^{2}}$, then

$$
H_{0}^{\perp} \subseteq \mathbb{C}\left(\alpha, H_{0}\right) \cap \mathbb{C}\left(\beta, H_{1}\right) .
$$

Proof. First we estimate the "angle" between $H_{0}$ and $H_{1}$. Since the cones $\mathbb{C}\left(\sqrt{1-\alpha^{2}}, H_{0}^{\perp}\right)$ and $\mathbb{C}\left(\sqrt{1-\beta^{2}}, H_{1}^{\perp}\right)$ have nonempty intersection they both must contain a common line $L \in G(n, 1)$.

$$
L \subseteq \mathbb{C}\left(\sqrt{1-\alpha^{2}}, H_{0}^{\perp}\right) \cap \mathbb{C}\left(\sqrt{1-\beta^{2}}, H_{1}^{\perp}\right) .
$$

Choose some point $z \in H_{1}$ and find a point $y \in L$ such that $z=\pi_{H_{1}}(y)$. Since $y \in$ $\mathbb{C}\left(\sqrt{1-\beta^{2}}, H_{1}^{\perp}\right)$ it follows that $\left|\pi_{H_{1}}^{\perp}(y)\right|<\beta|y|$. Furthermore, by the Pythagorean theorem

$$
|y|^{2}=\left|\pi_{H_{1}}(y)\right|^{2}+\left|\pi_{H_{1}}^{\perp}(y)\right|^{2} \leq|z|^{2}+\beta^{2}|y|^{2} ; \quad \text { hence } \quad|y| \leq \frac{|z|}{\sqrt{1-\beta^{2}}} .
$$

Because $y$ also belongs to the cone $\mathbb{C}\left(\sqrt{1-\alpha^{2}}, H_{0}^{\perp}\right)$ we have $\left|\pi_{H_{0}}^{\perp}(y)\right|<\alpha|y|$, so we obtain

$$
\begin{aligned}
\left|\pi_{H_{0}}^{\perp}(z)\right| & \leq\left|\pi_{H_{0}}^{\perp}(y)\right|+\left|\pi_{H_{0}}^{\perp}(z-y)\right| \leq\left|\pi_{H_{0}}^{\perp}(y)\right|+|z-y| \\
& =\left|\pi_{H_{0}}^{\perp}(y)\right|+\left|\pi_{H_{1}}^{\perp}(y)\right| \leq \alpha|y|+\beta|y| \leq \frac{\alpha+\beta}{\sqrt{1-\beta^{2}}}|z| \quad \text { for all } z \in H_{1} .
\end{aligned}
$$

Choose some $\epsilon>0$ and let

$$
x \in \mathbb{C}\left(\frac{\alpha+\beta}{\sqrt{1-\beta^{2}}}+\epsilon, H_{0}\right), \quad \text { so } \quad\left|\pi_{H_{0}}^{\perp}(x)\right| \geq\left(\frac{\alpha+\beta}{\sqrt{1-\beta^{2}}}+\epsilon\right)|x| .
$$

If $\epsilon$ is small enough, then such $x$ exists by the assumption that $\alpha+\beta<\sqrt{1-\beta^{2}}$. For bigger $\epsilon$ the inclusion $\mathbb{C}\left((\alpha+\beta) / \sqrt{1-\beta^{2}}+\epsilon, H_{0}\right) \subseteq \mathbb{C}\left(\epsilon, H_{1}\right)$ is trivially true. From the triangle inequality

$$
\begin{aligned}
& \frac{\alpha+\beta}{\sqrt{1-\beta^{2}}}|x| \leq\left|\pi_{H_{0}}^{\perp}(x)\right| \leq\left|\pi_{H_{0}}^{\perp}\left(\pi_{H_{1}}^{\perp}(x)\right)\right|+\left|\pi_{H_{0}}^{\perp}\left(\pi_{H_{1}}(x)\right)\right| \\
& \quad \leq\left|\pi_{H_{1}}^{\perp}(x)\right|+\left|\pi_{H_{0}}^{\perp}\left(\pi_{H_{1}}(x)\right)\right| ; \\
& \text { hence }\left|\pi_{H_{1}}^{\perp}(x)\right| \geq \frac{\alpha+\beta}{\sqrt{1-\beta^{2}}}|x|+\epsilon|x|-\left|\pi_{H_{0}}^{\perp}\left(\pi_{H_{1}}(x)\right)\right| .
\end{aligned}
$$

Because $\pi_{H_{1}}(x) \in H_{1}$ and because of estimate (17) we have

$$
\left|\pi_{H_{1}}^{\perp}(x)\right| \geq \frac{\alpha+\beta}{\sqrt{1-\beta^{2}}}|x|+\epsilon|x|-\frac{\alpha+\beta}{\sqrt{1-\beta^{2}}}\left|\pi_{H_{1}}(x)\right| \geq \epsilon|x| .
$$


3.3. The construction of voluminous simplices. For any $x_{0} \in \Sigma^{*}$ Proposition 3.18 stated below, ensures the existence of $d=d\left(x_{0}\right)>0$ and an $(\eta, d)$ voluminous simplex with vertices on $\Sigma \cap \overline{\mathbb{B}}\left(x_{0}, d\right)$ and also that at any scale below $d$ our set $\Sigma$ has big projection onto some affine $m$-plane. The reasoning used here mimics [26, Proposition 3.5]. Note that, finiteness of the $\mathcal{E}_{p}^{l}$-energy is not used in the proof.

Proposition 3.18. Let $\delta \in(0,1)$ and $\Sigma \in \mathcal{A}(\delta, m)$ be an admissible set. There exists an $\eta_{0}=\eta_{0}(\delta, m)>0$ such that for every point $x_{0} \in \Sigma^{*}$ there is a stopping distance $d=d\left(x_{0}\right)>0$ and an $(m+1)$-tuple of points $\left(x_{1}, x_{2}, \ldots, x_{m+1}\right) \in \Sigma^{m+1}$ such that $\mathbf{T}=\triangle\left(x_{0}, \ldots, x_{m+1}\right)$ is $\left(\eta_{0}, d\right)$-voluminous. Moreover, for all $\rho \in\left(0, \frac{1}{2} d\right)$ there exists an $m$-dimensional subspace $H=H\left(x_{0}, \rho\right) \in G(n, m)$ with the property

$$
\left(x_{0}+H\right) \cap \mathbb{B}\left(x_{0}, \sqrt{1-\delta^{2}} \rho\right) \subseteq \pi_{x_{0}+H}\left(\Sigma \cap \mathbb{B}\left(x_{0}, \rho\right)\right) .
$$

Corollary 3.19. For any $x_{0} \in \Sigma^{*}$ and any $\rho \leq \frac{1}{2} d\left(x_{0}\right)$ we have

$$
\mathcal{H}^{m}\left(\Sigma \cap \mathbb{B}\left(x_{0}, \rho\right)\right) \geq\left(1-\delta^{2}\right)^{\frac{m}{2}} \omega_{m} \rho^{m} .
$$

Proof. The orthogonal projection $\pi_{x_{0}+H}$ is Lipschitz with constant 1 so it cannot increase the $\mathcal{H}^{m}$-measure. From (18) we know that the image of $\Sigma \cap \mathbb{B}\left(x_{0}, \rho\right)$ under $\pi_{x_{0}+H}$ contains the ball $\left(x_{0}+H\right) \cap \mathbb{B}\left(x_{0}, \sqrt{1-\delta^{2}} \rho\right)$. The measure of that ball equals $\left(1-\delta^{2}\right)^{\frac{m}{2}} \omega_{m} \rho^{m}$.

Proof of Proposition 3.18. Without loss of generality we can assume that $x_{0}=0$ is the origin. To prove the proposition we will construct finite sequences of

- compact, connected, centrally symmetric sets $F_{0} \subseteq F_{1} \subseteq \ldots \subseteq F_{N}$,

- $m$-dimensional subspaces $H_{i} \subseteq \mathbb{R}^{n}$ for $i=0,1, \ldots, N$,

- and of radii $\rho_{0}<\rho_{1}<\cdots<\rho_{N}$.

For brevity, we define

$$
r_{i}=\sqrt{1-\delta^{2}} \rho_{i} .
$$

The above sequences will satisfy the following conditions:

- the interior of $F_{i}$ is disjoint with $\Sigma$, i.e.

$$
\Sigma \cap \operatorname{int} F_{i}=\emptyset
$$

- the radii grow geometrically, i.e.

$$
\rho_{i+1} \geq 2 \rho_{i},
$$

- for each $i \geq 0$ the set $F_{i+1}$ contains a large conical cap, i.e.

$$
\mathbb{C}\left(\delta, H_{i+1}, \frac{1}{2} \rho_{i}, \rho_{i+1}\right) \subseteq F_{i+1},
$$

- all spheres $S$ centered at $H_{i} \cap \mathbb{B}_{r_{i}}$, perpendicular to $H_{i}$ (i.e. $S \subseteq H_{i}^{\perp}+p$ for some $p \in \mathbb{R}^{n}$ ) and contained in $F_{i}$ are linked with $\Sigma$, i.e.

(22) $\forall x \in H_{i} \cap \mathbb{B}_{r_{i}} \forall s>0\left(S=\mathbb{S}(x, s) \cap\left(x+H_{i}^{\perp}\right) \subseteq F_{i} \quad \Rightarrow \quad \operatorname{lk}_{2}(\Sigma, S)=1\right)$.

Let us define the first elements of these sequences. We set $H_{0}=H_{x_{0}}, \rho_{0}=0$ and $F_{0}=\emptyset$. Next, we set

$$
H_{1}=H_{0}, \quad \rho_{1}=\inf \left\{s>0: \mathbb{C}\left(\delta, H_{0}, 0, s\right) \cap \Sigma \neq \emptyset\right\} \quad \text { and } \quad F_{1}=\mathbb{C}\left(\delta, H_{1}, 0, \rho_{1}\right) .
$$

Directly from the definition of an admissible set, we know that $\rho_{1}>0$, so the condition (20) is satisfied for $i=0$. Conditions (19) and (21) are immediate for $i=0$. Using Proposition 3.16 one can deform any sphere $S$ from condition (22) to 
the sphere $\mathscr{S}_{x}$ defined in $\mathrm{A2}$ of the definition of $\mathcal{A}(\delta, m)$. This shows that (22) is satisfied for $i=0$.

We proceed by induction. Assume we have already defined the sets $F_{i}$, subspaces $H_{i}$ and radii $\rho_{i}$ for $i=0,1, \ldots, I$. Now, we will show how to continue the construction.

Let $\left(e_{1}, e_{2}, \ldots, e_{m}\right)$ be an orthonormal basis of $H_{I}$. We choose $m$ points lying on $\Sigma$ such that

$$
x_{i} \in \Sigma \cap \mathbb{B}\left(r_{I} e_{i}, \delta \rho_{I}\right) \cap\left(H_{I}^{\perp}+r_{I} e_{i}\right)
$$

$$
\text { and in particular } x_{i} \in \mathbb{B}\left(x_{0}, 2 \rho_{I}\right) \text { for } i \in\{0,1, \ldots, m\} \text {. }
$$

Condition (22) together with Proposition 3.5 ensure that such points exist. The $m$-simplex $\mathbf{R}=\triangle\left(x_{0}, x_{1}, \ldots, x_{m}\right)$ will be the base of our $(m+1)$-simplex $\mathbf{T}$. Note that

$$
\begin{gathered}
\operatorname{diam}(\mathbf{R}) \leq 4 \rho_{I} \quad \text { and } \quad \pi_{H_{I}}(\mathbf{R})=\triangle\left(0, r_{I} e_{1}, r_{I} e_{2}, \ldots, r_{I} e_{m}\right) ; \\
\text { hence } \quad \mathcal{H}^{m}(\mathbf{R}) \geq \frac{r_{I}^{m}}{m !} .
\end{gathered}
$$

Recall that $x_{0}=0$ and set $P=\operatorname{span}\left\{x_{1}, x_{2}, \ldots, x_{m}\right\}$. It suffices to find one more point $x_{m+1} \in \Sigma$ such that the distance $\operatorname{dist}\left(x_{m+1}, P\right) \geq \tilde{\eta} \rho_{I}$ for some positive $\tilde{\eta}$. Indeed, if we set $\mathbf{T}=\triangle\left(x_{0}, \ldots, x_{m+1}\right)$, we have

$$
\mathfrak{h}_{\min }(\mathbf{T})=\frac{(m+1) \mathcal{H}^{m+1}(\mathbf{T})}{\max \left\{\mathcal{H}^{m}\left(\mathfrak{f}_{i} \mathbf{T}\right)\right\}_{i=0}^{m+1}} \geq \frac{\tilde{\eta} \rho_{I}(m+1) \mathcal{H}^{m}(\mathbf{R})}{\left(4 \rho_{I}\right)^{m} \omega_{m}} \geq\left(4 \rho_{I}\right) \frac{\tilde{\eta}\left(1-\delta^{2}\right)^{\frac{m}{2}}}{\omega_{m} 4^{m+1} m !}
$$

Choose a small positive number $h_{0}=h_{0}(\delta) \leq \frac{1}{2}$ such that

$$
\delta+2 h_{0} \delta \leq\left(1-2 h_{0} \delta\right) \sqrt{1-\left(2 h_{0} \delta\right)^{2}} .
$$

This is always possible because when we decrease $h_{0}$ to 0 the left-hand side of (25) converges to $\delta<1$ and the right-hand side converges to 1 . We need this condition to be able to apply Proposition 3.17 later on.

Remark 3.20. Note that if $\delta \leq \frac{1}{4}$, we can set $h_{0}=\frac{1}{2}$ because then

$$
\delta+2 h_{0} \delta \leq \frac{1}{2} \quad \text { and } \quad\left(1-2 h_{0} \delta\right) \sqrt{1-\left(2 h_{0} \delta\right)^{2}} \geq \frac{3}{4} \frac{\sqrt{15}}{16} \geq \frac{9}{16} .
$$

There are two possibilities (see Figure 4):

(A) there exists a point $x_{m+1} \in \Sigma \cap \mathbb{A}\left(\frac{1}{2} \rho_{I}, 2 \rho_{I}\right)$ such that

$$
\operatorname{dist}\left(x_{m+1}, P\right) \geq h_{0} \delta \rho_{I},
$$

(B) $\Sigma$ is contained in a small neighborhood of $P$, i.e.

$$
\Sigma \cap \mathbb{A}\left(\frac{1}{2} \rho_{I}, 2 \rho_{I}\right) \subseteq P+\mathbb{B}_{h_{0} \delta \rho_{I}} .
$$

If case (A) occurs, then we can end our construction immediately. The point $x_{m+1}$ satisfies

$$
x_{m+1} \in \mathbb{B}\left(x_{0}, 2 \rho_{I}\right) \text { and } \operatorname{dist}\left(x_{m+1}, P\right) \geq h_{0} \delta \rho_{I} .
$$

Hence, recalling (24), we may set

$$
\mathbf{T}=\triangle\left(x_{0}, \ldots, x_{m+1}\right), \quad N=I, \quad \eta_{0}=\frac{h_{0} \delta\left(1-\delta^{2}\right)^{\frac{m}{2}}}{\omega_{m} 4^{m+1} m !} \quad \text { and } \quad d=d\left(x_{0}\right)=4 \rho_{I} .
$$



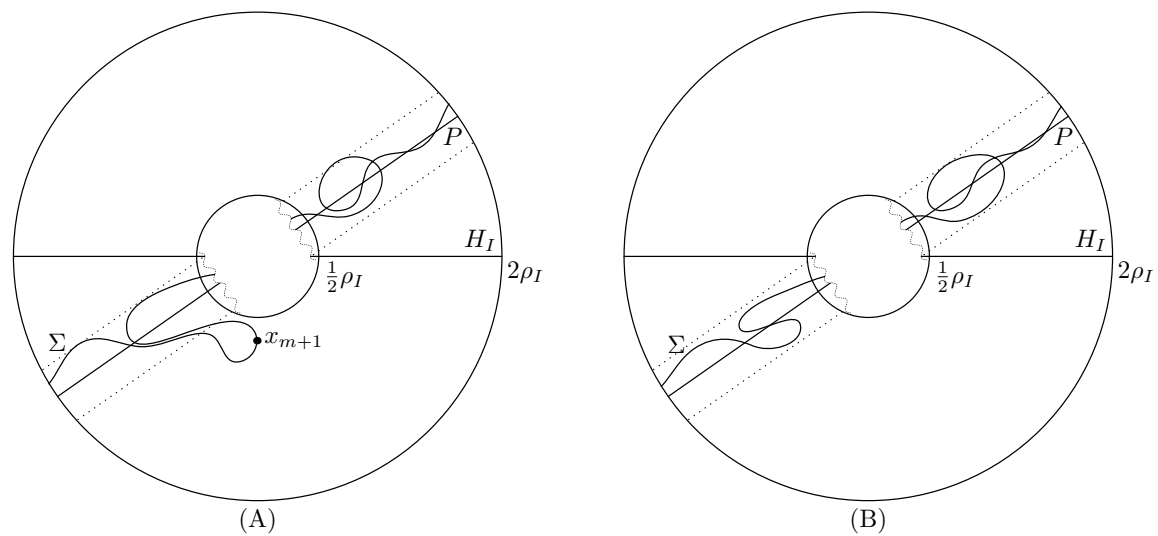

Figure 4. The two possible configurations.

If case (B) occurs, then our set $\Sigma$ is almost flat in $\mathbb{A}\left(\frac{1}{2} \rho_{I}, 2 \rho_{I}\right)$ so there is no chance of finding a voluminous simplex in this scale and we have to continue our construction. Let

- $H_{I+1}=P$,

- $\rho_{I+1}=\inf \left\{s>\rho_{I}: \mathbb{C}\left(\delta, P, \rho_{I}, s\right) \cap \Sigma \neq \emptyset\right\}$ and

- $F_{I+1}=F_{I} \cup \mathbb{C}\left(\delta, P, \frac{1}{2} \rho_{I}, \rho_{I+1}\right)$.

We assumed (B), so it follows that

$$
\forall x \in \Sigma \cap \mathbb{A}\left(\frac{1}{2} \rho_{I}, 2 \rho_{I}\right) \quad\left|\pi_{P}^{\perp}(x)\right| \leq h_{0} \delta \rho_{I} \leq 2 h_{0} \delta|x|<\delta|x| .
$$

This means that $\mathbb{C}\left(\delta, P, \frac{1}{2} \rho_{I}, 2 \rho_{I}\right)$ does not intersect $\Sigma$ and we can safely set $H_{I+1}=$ $P$. It is immediate that $\rho_{I+1} \geq 2 \rho_{I}$ so conditions (19), (20) and (21) are satisfied. Now, the only thing left is to verify condition (22).

We are going to show that all spheres $S$ contained in $F_{I+1}$ of the form

$$
S=\mathbb{S}(x, r) \cap\left(x+P^{\perp}\right), \quad \text { for some } x \in P \cap \mathbb{B}_{r_{I+1}}
$$

are linked with $\Sigma$. By the inductive assumption, we already know that spheres centered at $H_{I} \cap \mathbb{B}_{r_{I}}$, perpendicular to $H_{I}$ and contained in $F_{I}$ are linked with $\Sigma$. Therefore, all we need to do is to continuously deform $S$ to an appropriate sphere centered at $H_{I}$ and contained in $F_{I}$ in such a way that we never leave the set $F_{I+1}$ (see Figure 5).

We know that $F_{I+1}$ contains the conical cap $\mathbf{C}=\mathbb{C}\left(\delta, P, \frac{1}{2} \rho_{I}, \rho_{I+1}\right)$, so we can use Proposition 3.16 to move $S$ inside $\mathbf{C}$, so that it is centered at the origin.

From (27) we get

$$
\Sigma \cap \mathbb{A}\left(\frac{1}{2} \rho_{I}, 2 \rho_{I}\right) \subseteq \mathbb{R}^{n} \backslash \mathbb{C}\left(2 h_{0} \delta, P\right) \subseteq \mathbb{C}\left(\sqrt{1-\left(2 h_{0} \delta\right)^{2}}, P^{\perp}\right) .
$$

Using this and our inductive assumption we obtain

$$
\Sigma \cap \mathbb{A}\left(\frac{1}{2} \rho_{I}, \rho_{I}\right) \subseteq \mathbb{C}\left(\sqrt{1-\delta^{2}}, H_{I}^{\perp}\right) \cap \mathbb{C}\left(\sqrt{1-\left(2 h_{0} \delta\right)^{2}}, P^{\perp}\right) .
$$

We have two cones that have nonempty intersection and we chose $h_{0}$ such that (25) holds, so we can apply Proposition 3.17 with $\alpha=\delta$ and $\beta=2 h_{0} \delta$. Hence the intersection $\mathbb{C}\left(\delta, H_{I}\right) \cap \mathbb{C}(\delta, P)$ contains the space $H_{I}^{\perp}$. Therefore

$$
H_{I}^{\perp} \cap \mathbb{A}\left(\frac{1}{2} \rho_{I}, \rho_{I+1}\right) \subseteq \mathbb{C}\left(\delta, P, \frac{1}{2} \rho_{I}, \rho_{I+1}\right) \cap F_{I} .
$$




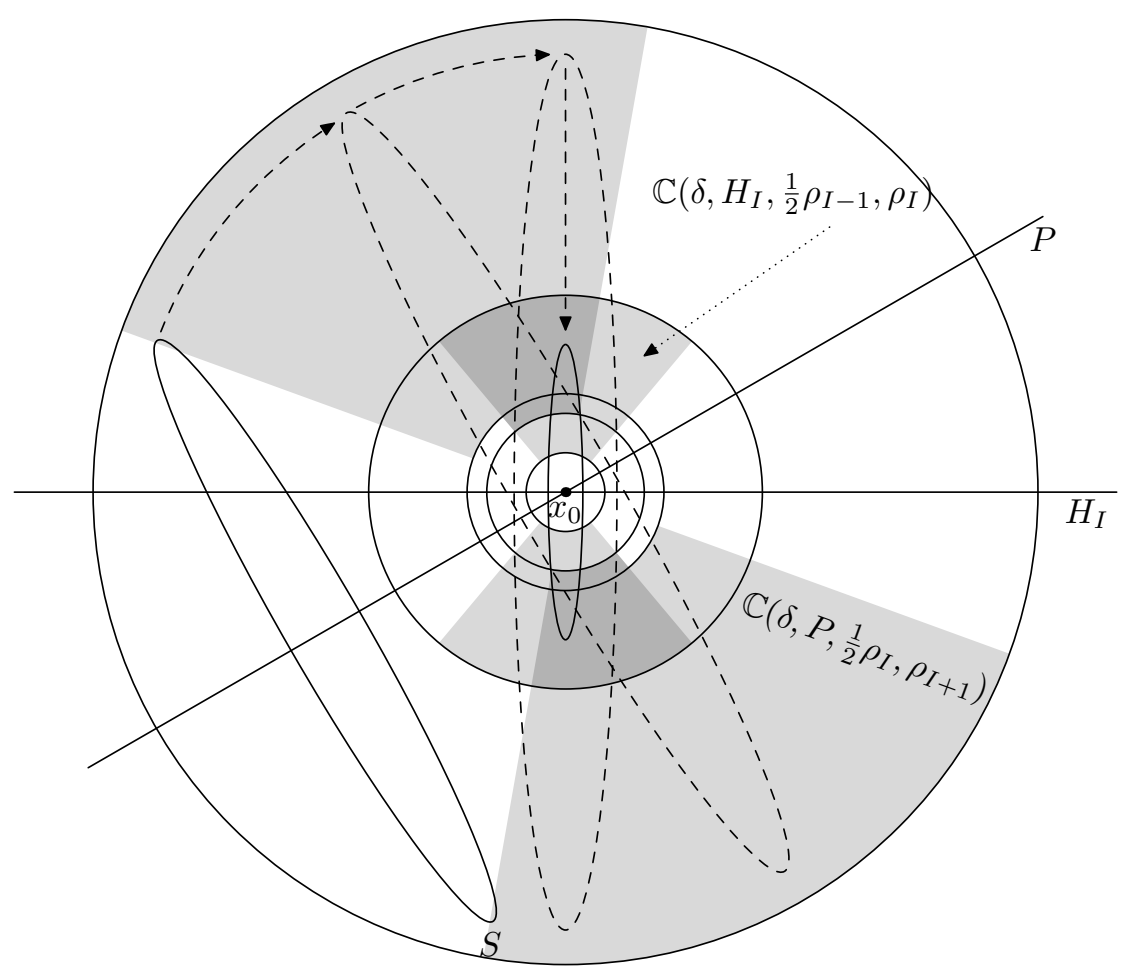

Figure 5. First we move the center of $S$ to $x_{0}$. Then we rotate $S$ so that it is perpendicular to $H_{I}$. Finally we change the radius so that it is between $\frac{1}{2} \rho_{I-1}$ and $\rho_{I}$.

Using Corollary 3.15] we can rotate $S$ inside $\mathbf{C}$, so that it lies in $H^{\perp}$. Then we decrease the radius of $S$ to the value e.g. $\frac{3}{4} \rho_{I} \in\left(\frac{1}{2} \rho_{I-1}, \rho_{I}\right)$. Applying the inductive assumption we obtain condition (22) for $i=I+1$.

The set $\Sigma$ is compact and $\rho_{i}$ grows geometrically, so our construction has to end eventually. Otherwise we would find arbitrary large spheres, which are linked with $\Sigma$ but this contradicts compactness.

\subsection{The proof of Theorem 2 ,}

Proof of Theorem 2. From Theorem 1 we already know that $\Sigma$ is an embedded, $C^{1, \lambda / \kappa}$-smooth manifold without boundary. Hence, it is also $(\delta, m)$-admissible for any $\delta \in(0,1)$ (cf. Example 3.6) and $\Sigma^{*}=\Sigma$. Set $\delta=\frac{1}{4}$, then Corollary 3.19 gives us Theorem 2 where $R_{0}$ can be any number less than $d(\Sigma)=\inf _{x_{0} \in \Sigma} d\left(x_{0}\right)$. Hence, it suffices to show that $d(\Sigma)$ can be bounded below independently of $\Sigma$.

From Proposition 2.1 we know that $d(\Sigma)$ must satisfy (11) with $\eta=\eta_{0}$ defined by (26). Hence, we already have a positive lower bound on $d(\Sigma)$. We only need to show that it does not depend on $A_{\mathrm{Ahl}}$. 
Fix a point $x_{0} \in \Sigma$ such that $d\left(x_{0}\right)<(1+\varepsilon) d(\Sigma)$ for some small $\varepsilon \in(0,1)$. Proposition 3.18 gives us an $\left(\eta_{0}, d\left(x_{0}\right)\right)$-voluminous simplex $\triangle\left(x_{0}, \ldots, x_{m+1}\right)$. Recall that $\varsigma_{m+1}<\frac{1}{4}$ was defined by (2). For each $i=1,2, \ldots, m+1$ we have

$$
\varsigma_{m+1} d\left(x_{0}\right) \leq \varsigma_{m+1}(1+\varepsilon) d(\Sigma) \leq \frac{1}{2} d(\Sigma) \leq \frac{1}{2} d\left(x_{i}\right) .
$$

Hence, applying Corollary 3.19 we get

$$
\mathcal{H}^{m}\left(\Sigma \cap \mathbb{B}\left(x_{i}, \varsigma_{m+1} d\left(x_{0}\right)\right)\right) \geq \frac{\sqrt{15^{m}}}{4^{m}} \omega_{m}\left(\varsigma_{m+1} d\left(x_{0}\right)\right)^{m} .
$$

Now we can repeat the calculation from the proof of Proposition 2.1, replacing $A_{\mathrm{Ahl}}$ with $\frac{\sqrt{15^{m}}}{4^{m}} \omega_{m}$, to obtain

$$
E \geq\left(\frac{\sqrt{15^{m}}}{4^{m}} \omega_{m}\left(\varsigma_{m+1} d\left(x_{0}\right)\right)^{m}\right)^{l}\left(\frac{3 \eta_{0}^{m+1}}{4(m+1) ! d\left(x_{0}\right)}\right)^{p}=C(m, l, p) d\left(x_{0}\right)^{m l-p} .
$$

Therefore

$$
\frac{1}{2} d(\Sigma)=\frac{1}{2} \lim _{\varepsilon \rightarrow 0^{+}}(1+\varepsilon) d(\Sigma) \geq \frac{1}{2} d\left(x_{0}\right) \geq C(m, l, p) E^{\frac{-1}{\lambda}}=R_{0} .
$$

3.5. Removing the dependence on $M_{\theta \beta}$ and $R_{\theta \beta}$. In this section we show that if $\Sigma$ is $m$-fine with finite $\mathcal{E}_{p}^{l}$-energy, then the constants $M_{\theta \beta}$ and $R_{\theta \beta}$ from Theorem 1 can be chosen depending solely on $E, m, l$ and $p$.

Proposition 3.21. Let $\Sigma \subseteq \mathbb{R}^{n}$ be an $m$-fine set such that $\mathcal{E}_{p}^{l}(\Sigma) \leq E<\infty$ for some $p>m l$. Then there exists $R_{1}=R_{1}(E, m, l, p)$ such that $\Sigma$ satisfies (Ah]) and $\theta \lesssim \beta$ with constants $M_{\theta \beta}=5, R_{\theta \beta}=R_{\mathrm{Ahl}}=R_{1}$ and $A_{\mathrm{Ahl}}=\frac{\sqrt{15^{m}}}{4^{m}} \omega_{m}$.

Proof. From Theorem 1 and Theorem 2 we already know that $\Sigma$ is $\left(\frac{1}{4}, m\right)$-admissible with $\Sigma^{*}=\Sigma$ and satisfies (Ah]) with $R_{\mathrm{Ahl}}=R_{0}$ and $A_{\mathrm{Ahl}}=\frac{\sqrt{15^{m}}}{4^{m}} \omega_{m}$. Hence, by Proposition 1] we also have

$$
\forall r \leq R_{0} \forall x \in \Sigma \quad \beta_{m}^{\Sigma}(x, r) \leq C(m, l, p) E^{\frac{1}{\kappa}} r^{\frac{\lambda}{\kappa}} .
$$

Fix a point $x_{0} \in \Sigma$ and a radius $r \leq R_{0}$. Choose some $m$-plane $P \in G(n, m)$ such that

$$
\forall y \in \Sigma \cap \overline{\mathbb{B}}\left(x_{0}, r\right) \quad\left|\pi_{P}^{\perp}\left(y-x_{0}\right)\right| \leq \beta_{m}^{\Sigma}\left(x_{0}, r\right) r .
$$

For brevity we set $\beta=\beta_{m}^{\Sigma}\left(x_{0}, r\right)$ and $\gamma=\frac{\sqrt{15}}{4}$. Inspecting the proof of Proposition 3.18 we can find $i \in \mathbb{N}$ such that $\rho_{i} \leq r<\rho_{i+1}$. We set $H=H_{i}$. Let $y \in \mathbb{R}^{n}$ be any point such that $y-x_{0} \in H$ and $\left|y-x_{0}\right|=\gamma r$. We see that $\mathbb{S}\left(y, \frac{1}{4} r\right) \cap\left(y+H^{\perp}\right)$ is linked with $\Sigma$; hence (cf. Proposition 3.5) there exists $z \in \Sigma \cap \mathbb{B}\left(y, \frac{1}{4} r\right) \cap\left(y+H^{\perp}\right)$. Note that $\gamma r \leq\left|z-x_{0}\right| \leq r$, so

$$
\frac{\left|\pi \frac{\perp}{P}\left(z-x_{0}\right)\right|}{\left|z-x_{0}\right|} \leq \frac{\beta r}{\gamma r}=\frac{\beta}{\gamma} ; \quad \text { hence } \quad\left(z-x_{0}\right) \in \mathbb{C}\left(\left(1-\frac{\beta^{2}}{\gamma^{2}}\right)^{\frac{1}{2}}, P^{\perp}\right) \cap \mathbb{C}\left(\gamma, H^{\perp}\right) .
$$

To apply Proposition 3.17 we need to ensure the condition (30)

$$
\sqrt{1-\gamma^{2}}+\frac{\beta}{\gamma} \leq\left(1-\frac{\beta}{\gamma}\right) \sqrt{1-\left(\frac{\beta}{\gamma}\right)^{2}} \Longleftrightarrow \frac{\beta}{\gamma} \leq\left(1-\frac{\beta}{\gamma}\right) \sqrt{1-\left(\frac{\beta}{\gamma}\right)^{2}}-\sqrt{1-\gamma^{2}} .
$$


Substituting $\Psi=\frac{\beta}{\gamma}$ in (30) and recalling that $\gamma=\frac{\sqrt{15}}{4}$ we obtain the following inequality:

$$
\Psi \leq(1-\Psi) \sqrt{1-\Psi^{2}}-\frac{1}{4} .
$$

Note that if $\Psi \rightarrow 0$, then the right-hand side converges to $\frac{3}{4}$. Let $\Psi_{0}$ be the smallest, positive root of the equation $\Psi=(1-\Psi) \sqrt{1-\Psi^{2}}-\frac{1}{4}$. Then any $\Psi \in\left[0, \Psi_{0}\right]$ satisfies (31). Recall that $\beta=\beta_{m}^{\Sigma}(x, r) \leq C(m, l, p) E^{1 / \kappa} r^{\lambda / \kappa}$, so to ensure condition (30) it suffices to impose the following constraint:

$$
r \leq \min \left\{\frac{1}{2}\left(\frac{\gamma \Psi_{0}}{C(m, l, p)}\right)^{\frac{\kappa}{\lambda}} E^{\frac{-1}{\lambda}}, R_{0}\right\}=R_{1}(E, m, l, p) .
$$

Now, for such $r$ we can use Proposition 3.17 to obtain

$$
H^{\perp} \subseteq \mathbb{C}\left(\frac{1}{4}, H\right) \cap \mathbb{C}\left(\frac{\beta}{\gamma}, P\right) .
$$

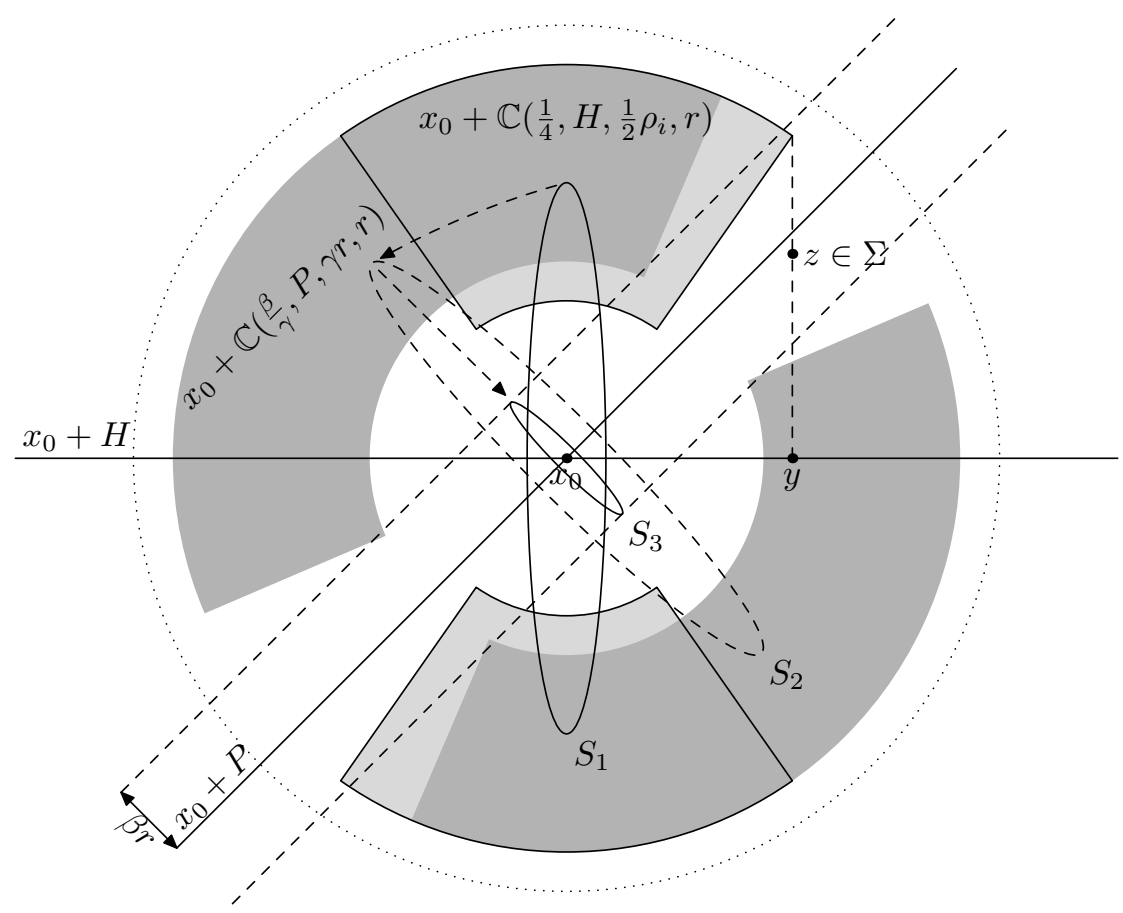

Figure 6 . If $\beta$ is small enough, then the cone $\mathbb{C}\left(\frac{8 \beta}{7 \gamma}, P\right)$ contains $H^{\perp}$ and we can continuously transform $S_{1}$ into $S_{3}$ inside the conical cap $\mathbb{C}\left(\frac{8 \beta}{7 \gamma}, P, \frac{7}{8} r \gamma, \frac{7}{8} r\right)$.

We set $\mathbf{C}=x_{0}+\mathbb{C}\left(\frac{1}{4}, H, \frac{1}{2} \rho_{i}, \rho_{i+1}\right)$ and $S_{1}=\mathbb{S}\left(x_{0}, r\right) \cap\left(x_{0}+H^{\perp}\right) \subseteq \mathbf{C}$. Observe that $\mathbf{C} \cap \Sigma=\emptyset$ and $\operatorname{lk}_{2}\left(S_{1}, \Sigma\right)=1$. Using Corollary 3.15 we rotate $S_{1}$ into $S_{2}=$ $\mathbb{S}\left(x_{0}, r\right) \cap\left(x_{0}+P^{\perp}\right)$ (see Figure 6) inside $\mathbb{C}\left(\frac{\beta}{\gamma}, P, r \gamma, r\right)$. Note that for $x \in \Sigma$ such that $\left|x-x_{0}\right|>\gamma r$ we have

$$
\frac{\pi_{P}\left(x-x_{0}\right)}{\left|x-x_{0}\right|}<\frac{\beta r}{\gamma r}=\frac{\beta}{\gamma}
$$


hence the conical cap $\mathbb{C}\left(\frac{\beta}{\gamma}, P, \gamma r, r\right)$ does not intersect $\Sigma$ and the resulting sphere $S_{2}$ is still linked with $\Sigma$. Next we decrease the radius of $S_{2}$ to the value $\beta r$ obtaining another sphere $S_{3}=\mathbb{S}\left(x_{0}, \beta r\right) \cap\left(x_{0}+P^{\perp}\right)$ which is also linked with $\Sigma$.

We can translate $S_{3}$ along any vector $v \in P$ with $|v| \leq \sqrt{1-\beta^{2}} r$ without changing the linking number. This way we see that for any point $w \in\left(x_{0}+P\right) \cap$ $\overline{\mathbb{B}}\left(x_{0}, \sqrt{1-\beta^{2}} r\right)$ there exists a point $z \in \Sigma$ such that $|z-w| \leq \beta r$.

For any other point $w \in\left(x_{0}+P\right)$ with $\sqrt{1-\beta^{2}} r \leq\left|w-x_{0}\right| \leq r$ we set

$$
\tilde{w}=w-\left(w-x_{0}\right)\left|w-x_{0}\right|^{-1}\left(1-\sqrt{1-\beta^{2}}\right) r,
$$

so that $\left|\tilde{w}-x_{0}\right| \leq \sqrt{1-\beta^{2}} r$. Then we find $z \in \Sigma$ such that $|\tilde{w}-z| \leq \beta r$ and we obtain the estimate

$$
\begin{aligned}
|z-w| & \leq|z-\tilde{w}|+|\tilde{w}-w| \leq \beta r+\left(1-\sqrt{1-\beta^{2}}\right) r \\
& =r\left(\beta+\frac{\beta^{2}}{1+\sqrt{1-\beta^{2}}}\right) \leq 2 \beta r=4 \beta_{m}^{\Sigma}(x, r) r .
\end{aligned}
$$

This implies that $d_{\mathcal{H}}\left(\Sigma \cap \overline{\mathbb{B}}\left(x_{0}, r\right),\left(x_{0}+P\right) \cap \overline{\mathbb{B}}\left(x_{0}, r\right)\right) \leq 5 \beta_{m}^{\Sigma}\left(x_{0}, r\right)$. Therefore the infimum over all $H \in G(n, m)$ must be even smaller, so $\theta_{m}^{\Sigma}\left(x_{0}, r\right) \leq 5 \beta_{m}^{\Sigma}\left(x_{0}, r\right)$ for any $r \leq R_{\theta \beta}=R_{1}$ and we can safely set $M_{\theta \beta}=5$.

\section{UNIFORM ESTIMATES ON THE LOCAL GRAPH REPRESENTATIONS}

For the sake of brevity we introduce the following notation

$$
\pi_{x}=\pi_{T_{x} \Sigma} \quad \text { and } \quad \pi_{x}^{\perp}=\pi_{T_{x} \Sigma}^{\perp},
$$

where $x \in \Sigma$. The main result of this section is

Theorem 4.1. Let $\Sigma \subseteq \mathbb{R}^{n}$ be an m-fine set. If $\mathcal{E}_{p}^{l}(\Sigma) \leq E<\infty$ for some $p>m l$, then $\Sigma$ is a closed $C^{1, \lambda / \kappa}$-manifold (by Theorem 11) and there exist constants $R_{\lambda \kappa}=R_{\lambda \kappa}(E, m, l, p)$ and $C_{\lambda \kappa}=C_{\lambda \kappa}(E, m, l, p) \geq 1$ such that for all $x \in \Sigma$ there exists a function $F_{x}: T_{x} \Sigma \rightarrow\left(T_{x} \Sigma\right)^{\perp}$ of class $C^{1, \lambda / \kappa}$ such that

$$
(\Sigma-x) \cap \mathbb{B}_{R_{\lambda \kappa}}=\operatorname{Graph}\left(F_{x}\right) \cap \mathbb{B}_{R_{\lambda \kappa}}
$$

$$
\text { and } \forall y, z \in T_{x} \Sigma \quad\left\|D F_{x}(y)-D F_{x}(z)\right\| \leq C_{\lambda \kappa}|y-z|^{\frac{\lambda}{\kappa}} .
$$

To prove this theorem we fix a point $x \in \Sigma$ and for each radius $r>0$ we choose the best approximating $m$-plane $P(x, r)$ for $\Sigma$ in $\overline{\mathbb{B}}(x, r)$. Then we use the fact that $\theta_{m}^{\Sigma}(x, r) \leq M_{\theta \beta} \beta_{m}^{\Sigma}(x, r)$ together with Proposition 1 to show that $P(x, r)$ converge to the tangent plane $T_{x} \Sigma$, when $r \rightarrow 0$. This also gives a bound on the oscillation of $T_{x} \Sigma$. Then we derive Lemma 4.8, which says that at some small scale we cannot have two distinct points $y$ and $z$ of $\Sigma$ such that the vector $v=(y-z)$ is orthogonal to $T_{x} \Sigma$. Any such vector $v$ would be close to the tangent plane $T_{z} \Sigma$ and this would violate the bound on the oscillation of tangent planes proved earlier. From here, it follows that there exists a small radius $R_{\lambda \kappa}$ such that $\Sigma \cap \mathbb{B}\left(x, R_{\lambda \kappa}\right)$ is a graph of some function $F_{x}$, which is of class $C^{1, \lambda / \kappa}$ by Theorem 1

In the sequel of this section we always assume that $\Sigma$ satisfies the hypotheses of Theorem 4.1 
4.1. Estimates on the oscillation of tangent planes. Combining Propositions 3.21 and 1 we see that

$$
\forall r \leq R_{1} \forall x \in \Sigma \quad \theta_{m}^{\Sigma}(x, r) \leq 5 \beta_{m}^{\Sigma}(x, r) \leq 5 C(m, l, p) E^{\frac{1}{\kappa}} r^{\frac{\lambda}{\kappa}} .
$$

Let $\tilde{R}_{1}=\tilde{R}_{1}(E, m, l, p) \in\left(0, R_{1}\right]$ be such that $5 C(m, l, p) E^{\frac{1}{\kappa}} r^{\frac{\lambda}{\kappa}} \leq \frac{1}{4}$ for all $r \leq \tilde{R}_{1}$, so $\tilde{R}_{1}=C_{0} E^{-1 / \lambda}$ for some $C_{0}=C_{0}(m, l, p)$.

Lemma 4.2. Choose a point $x \in \Sigma$ and fix some $r_{0} \leq \tilde{R}_{1}$. Choose another point $y \in \Sigma \cap \overline{\mathbb{B}}\left(x, \frac{1}{2} r_{0}\right)$ and some $r_{1} \in\left[\frac{1}{2} r_{0}, r_{0}-|x-y|\right]$. Let $H_{0} \in \operatorname{BAP}_{\mathrm{m}}\left(x, r_{0}\right)$ and $H_{1} \in \operatorname{BAP}_{\mathrm{m}}\left(y, r_{1}\right)$. Then there exists a constant $C_{h h}=C_{h h}(m, l, p) \geq 1$ such that

$$
d_{\mathrm{Gr}}\left(H_{0}, H_{1}\right) \leq C_{h h} E^{1 / \kappa} r_{0}^{\lambda / \kappa} .
$$

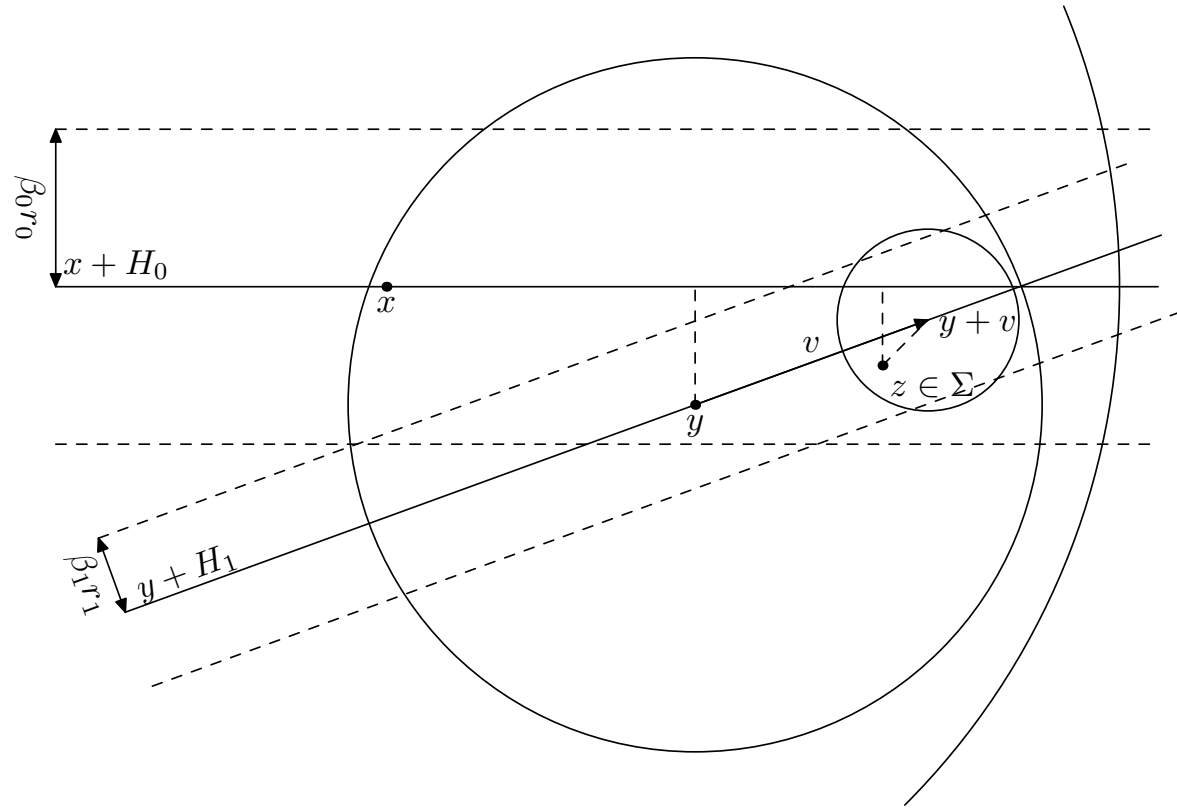

FiguRE 7. Existence of $z \in \Sigma$ is guaranteed by the condition $\theta \lesssim \beta$. This allows us to estimate $d_{\mathrm{Gr}}\left(H_{0}, H_{1}\right)$.

Proof. Set $\beta_{0}=\beta_{m}^{\Sigma}\left(x, r_{0}\right)$ and $\beta_{1}=\beta_{m}^{\Sigma}\left(y, r_{1}\right)$. Recall (33) to see that since $r_{1} \leq \tilde{R}_{1}$, we have $5 \beta_{1} \leq \frac{1}{4}$. Let $v \in H_{1}$ be any vector of length $|v|=r_{1}\left(1-5 \beta_{1}\right)$. Since $\theta_{m}^{\Sigma}\left(y, r_{1}\right) \leq 5 \beta_{1}$, there exists a point $z \in \Sigma \cap \overline{\mathbb{B}}\left(y+v, 5 \beta_{1} r_{1}\right)$. Hence $|(y+v)-z| \leq$ $5 \beta_{1} r_{1}$ (see Figure 7). Note that $\overline{\mathbb{B}}\left(y+v, 5 \beta_{1} r_{1}\right) \subseteq \overline{\mathbb{B}}\left(y, r_{1}\right) \subseteq \overline{\mathbb{B}}\left(x, r_{0}\right)$. Therefore $\operatorname{dist}\left(z, x+H_{0}\right)=\left|\pi_{H_{0}}^{\perp}(z-x)\right| \leq \beta_{0} r_{0}$ and we obtain the estimate

$$
\begin{aligned}
\left|\pi_{H_{0}}^{\perp}(v)\right| & \leq\left|\pi_{H_{0}}^{\perp}((y-x)+v)\right|+\left|\pi_{H_{0}}^{\perp}(y-x)\right| \\
& \leq|((y-x)+v)-(z-x)|+\left|\pi_{H_{0}}^{\perp}(z-x)\right|+\left|\pi_{H_{0}}^{\perp}(y-x)\right| \\
& \leq 5 \beta_{1} r_{1}+\beta_{0} r_{0}+\beta_{0} r_{0} \leq 7 C E^{1 / \kappa} r_{0}^{1+\lambda / \kappa} .
\end{aligned}
$$


Since $v$ was chosen arbitrarily we get the following estimate for any unit vector $e \in H_{1} \cap \mathbb{S}:$

$$
\left|\pi_{H_{0}}^{\perp}(e)\right| \leq 7 C E^{1 / \kappa} \frac{r_{0}^{1+\lambda / \kappa}}{r_{1}\left(1-5 \beta_{1}\right)} \leq 7 C E^{1 / \kappa} \frac{4 r_{0}^{1+\lambda / \kappa}}{3 r_{1}} .
$$

Recall that $r_{1} \geq \frac{1}{2} r_{0}$, so if $\left(e_{1}, \ldots, e_{m}\right)$ is any orthonormal basis of $H_{1}$, then for all $i=1, \ldots, m$ we have $\left|\pi_{H_{0}}^{\perp}\left(e_{i}\right)\right| \leq \frac{8 \cdot 7}{3} C E^{1 / \kappa} r_{0}^{\lambda / \kappa}$. Applying Lemma 1.18 we get

$$
d_{\mathrm{Gr}}\left(H_{0}, H_{1}\right) \leq \tilde{C}(m, l, p) C_{\pi}(m) E^{1 / \kappa} r_{0}^{\lambda / \kappa} .
$$

Lemma 4.3. Choose a point $x \in \Sigma$. For each $r \leq \tilde{R}_{1}$ fix an $m$-plane $P(r) \in$ $\operatorname{BAP}_{\mathrm{m}}(x, r)$. There exists a limit $\lim _{r \rightarrow 0} P(r)=T_{x} \Sigma \in G(n, m)$ and it does not depend on the choice of $P(r) \in \mathrm{BAP}_{\mathrm{m}}(x, r)$.

Proof. Set $\rho_{k}=2^{-k} \tilde{R}_{1}$ and for each $k$ choose $P_{k} \in \operatorname{BAP}_{\mathrm{m}}\left(x, \rho_{k}\right)$. Set $\beta_{k}=$ $\beta_{m}^{\Sigma}\left(x, \rho_{k}\right)$. We will show that $\{P(r)\}_{r<\tilde{R}_{1}}$ satisfies the Cauchy condition. Fix some $0<s<t<\rho_{0}$ and find two natural numbers $a<b$ such that $\rho_{b+1}<s \leq \rho_{b}$ and $\rho_{a+1}<t \leq \rho_{a}$.

Applying Lemma 4.2 with $x=y, r_{0}=\rho_{j}$ and $r_{1}=\frac{1}{2} r_{0}=\rho_{j+1}$ we obtain

$$
d_{\mathrm{Gr}}\left(P_{j}, P_{j+1}\right) \leq C E^{1 / \kappa} \rho_{j}^{\lambda / \kappa} .
$$

Setting $r_{0}=\rho_{b}$ and $r_{1}=s$ or $r_{0}=\rho_{a}$ and $r_{1}=t$ we also get

$$
d_{\mathrm{Gr}}\left(P(s), P_{b}\right) \leq C E^{1 / \kappa} \rho_{b}^{\lambda / \kappa} \quad \text { and } \quad d_{\mathrm{Gr}}\left(P(t), P_{a}\right) \leq C E^{1 / \kappa} \rho_{a}^{\lambda / \kappa} .
$$

Using these estimates we can write

$$
\begin{aligned}
d_{\mathrm{Gr}}(P(r), P(s)) & \leq d_{\mathrm{Gr}}\left(P(r), P_{a}\right)+\sum_{j=a}^{b-1} d_{\mathrm{Gr}}\left(P_{j}, P_{j+1}\right)+d_{\mathrm{Gr}}\left(P_{b}, P(s)\right) \\
& \leq C E^{1 / \kappa}\left(\rho_{a}^{\lambda / \kappa}+\sum_{j=a}^{b} \rho_{j}^{\lambda / \kappa}\right) \\
& =C E^{1 / \kappa} \rho_{a}^{\lambda / \kappa}\left(1+\sum_{j=0}^{b-a} 2^{-j \lambda / \kappa}\right) \leq \hat{C}(m, l, p) E^{1 / \kappa} \rho_{a}^{\lambda / \kappa}
\end{aligned}
$$

which shows that the Cauchy condition is satisfied, so $P(r)$ converges in $G(n, m)$ to some $m$-plane, which must be the tangent plane $T_{x} \Sigma$.

Corollary 4.4. There exists a constant $C_{t h}=C_{t h}(m, l, p) \geq 1$ such that for all $x \in \Sigma$, all $r \leq \tilde{R}_{1}$ and all $H \in \mathrm{BAP}_{\mathrm{m}}(x, r)$ we have

$$
d_{\mathrm{Gr}}\left(T_{x} \Sigma, H\right) \leq C_{t h} E^{1 / \kappa} r^{\lambda / \kappa} .
$$

Corollary 4.5. There exists a constant $C_{t p}=C_{t p}(m, l, p) \geq 1$ such that for all $x \in \Sigma$ and all $y \in \Sigma \cap \overline{\mathbb{B}}\left(x, \tilde{R}_{1}\right)$ we have

$$
\operatorname{dist}\left(y, x+T_{x} \Sigma\right)=\left|\pi_{x}^{\perp}(y-x)\right| \leq C_{t p} E^{1 / \kappa}|y-x|^{1+\lambda / \kappa} .
$$


Proof. Choose an $m$-plane $H \in \mathrm{BAP}_{\mathrm{m}}(x,|y-x|)$. Using (33) and Corollary 4.4 we get

$$
\begin{aligned}
\left|\pi_{x}^{\perp}(y-x)\right| & \leq\left|\pi_{H}^{\perp}(y-x)\right|+\left|\pi_{x}^{\perp}\left(\pi_{H}(y-x)\right)\right| \\
& \leq|y-x| \beta_{m}^{\Sigma}(x,|y-x|)+|y-x| C_{t h} E^{1 / \kappa}|y-x|^{\lambda / \kappa} \\
& \leq C_{t p} E^{1 / \kappa}|y-x|^{1+\lambda / \kappa} .
\end{aligned}
$$

Lemma 4.6. There exists a constant $C_{t t}=C_{t t}(m, l, p) \geq 1$ such that for all $x \in \Sigma$ and for all $y \in \Sigma \cap \overline{\mathbb{B}}\left(x, \frac{1}{2} \tilde{R}_{1}\right)$ we have

$$
d_{\mathrm{Gr}}\left(T_{x} \Sigma, T_{y} \Sigma\right) \leq C_{t t} E^{1 / \kappa}|x-y|^{\lambda / \kappa} .
$$

Proof. Let $y \in \Sigma \cap \bar{B}\left(x, \frac{1}{2} \tilde{R}_{1}\right)$. Set $r_{0}=2|x-y|$ and $r_{1}=|x-y|$. Choose any $H_{0} \in \operatorname{BAP}_{\mathrm{m}}\left(x, r_{0}\right)$ and any $H_{1} \in \operatorname{BAP}_{\mathrm{m}}\left(y, r_{1}\right)$. From Lemma 4.2 we have

$$
d_{\mathrm{Gr}}\left(H_{0}, H_{1}\right) \leq C E^{1 / \kappa} r_{0}^{\lambda / \kappa} .
$$

On the other hand Corollary 4.4 says that

$$
d_{\mathrm{Gr}}\left(T_{x} \Sigma, H_{0}\right) \leq C_{t h} E^{1 / \kappa} r_{0}^{\lambda / \kappa} \quad \text { and } \quad d_{\mathrm{Gr}}\left(T_{y} \Sigma, H_{1}\right) \leq C_{t h} E^{1 / \kappa} r_{0}^{\lambda / \kappa} .
$$

Putting these estimates together we obtain

$d_{\mathrm{Gr}}\left(T_{x} \Sigma, T_{y} \Sigma\right) \leq d_{\mathrm{Gr}}\left(T_{x} \Sigma, H_{0}\right)+d_{\mathrm{Gr}}\left(H_{0}, H_{1}\right)+d_{\mathrm{Gr}}\left(H_{1}, T_{y} \Sigma\right)=\bar{C} E^{1 / \kappa}|x-y|^{\lambda / \kappa}$.

4.2. Uniform estimates on the size of maps. Combining Corollary 4.5 and Lemma 4.6] one can see that if we have two distinct points $y, z \in \Sigma$ such that $y-z \perp T_{x} \Sigma$ and $|y-z| \lesssim|x-y|$, then the tangent plane $T_{y} \Sigma$ must form a large angle with the plane $T_{x} \Sigma$. Such situation can only happen far away from $x$ because of the bound on the oscillation of tangent planes.

Remark 4.7. Let $\iota=\iota(m)=\frac{\epsilon_{\rho \varepsilon}}{100}$. Lemma 4.6 allows us to find $\tilde{R}_{2} \in\left(0, \tilde{R}_{1}\right]$ such that $\tilde{R}_{2}=C(m, l, p) E^{-1 / \lambda}$ and whenever $|x-y| \leq \tilde{R}_{2}$ for some $x, y \in \Sigma$, then $d_{\mathrm{Gr}}\left(T_{x} \Sigma, T_{y} \Sigma\right) \leq \iota$.

Lemma 4.8. Choose any point $x \in \Sigma$. There exists a radius $R_{2}=C(m, l, p) E^{-1 / \lambda}$ $\in\left(0, \tilde{R}_{2}\right]$ such that if $y, z \in \Sigma \cap \overline{\mathbb{B}}\left(x, \frac{1}{2} \tilde{R}_{2}\right)$ and $(y-z) \perp T_{x} \Sigma$, then necessarily $\max \{|x-y|,|x-z|\}>R_{2}$.

Proof. Let $C_{t p} \geq 1$ be the constant from Corollary 4.5 and let $C_{0}$ be such that $\tilde{R}_{1}=C_{0} E^{-1 / \lambda}$ (cf. (33)). Assume there exist two points $y, z \in \Sigma \cap \bar{B}\left(x, \frac{1}{2} \tilde{R}_{2}\right)$ such that $(z-y) \perp T_{x} \Sigma$ and $\max \{|x-y|,|x-z|\} \leq \frac{1}{2} \tilde{R}_{1}\left(C_{0} C_{t p}\right)^{-1}$. Without loss of generality we can assume that $|x-z| \leq|x-y| \leq 1$; hence

$$
|x-z|^{1+\lambda / \kappa} \leq|x-y|^{1+\lambda / \kappa} \leq \tilde{R}_{1}^{\lambda / \kappa}|x-y| \leq C_{0} E^{-1 / \kappa}|x-y| .
$$

First we estimate the distance $|y-z|$ using Corollary 4.5.

$$
\begin{aligned}
|y-z| & =\left|\pi_{x}^{\perp}(y-z)\right| \leq\left|\pi_{x}^{\perp}(y-x)\right|+\left|\pi_{x}^{\perp}(x-z)\right| \\
& \leq C_{t p} E^{1 / \kappa}\left(|y-x|^{1+\lambda / \kappa}+|x-z|^{1+\lambda / \kappa}\right) \leq 2 C_{t p} C_{0}|x-y| \leq \tilde{R}_{1} .
\end{aligned}
$$


Hence we can use Corollary 4.5] once again to estimate the distance between $z$ and $T_{y} \Sigma$. Using the definition of $d_{\mathrm{Gr}}$ we may write

$$
\begin{aligned}
d_{\mathrm{Gr}}\left(T_{x} \Sigma, T_{y} \Sigma\right) & \geq|z-y|^{-1}\left|\pi_{x}(z-y)-\pi_{y}(z-y)\right|=|z-y|^{-1}\left|\pi_{y}(z-y)\right| \\
& \geq|z-y|^{-1}\left(|z-y|-\left|\pi_{y}^{\perp}(z-y)\right|\right) \\
& \geq|z-y|^{-1}\left(|z-y|-C_{t p} E^{1 / \kappa}|z-y|^{1+\lambda / \kappa}\right) \\
& =1-C_{t p} E^{1 / \kappa}|z-y|^{\lambda / \kappa} .
\end{aligned}
$$

On the other hand Lemma 4.6 gives us

$$
d_{\mathrm{Gr}}\left(T_{x} \Sigma, T_{y} \Sigma\right) \leq C_{t t} E^{1 / \kappa}|x-y|^{\lambda / \kappa} .
$$

Putting these two estimates together we have

$$
1-C_{t p} E^{1 / \kappa}|z-y|^{\lambda / \kappa} \leq d_{\mathrm{Gr}}\left(T_{x} \Sigma, T_{y} \Sigma\right) \leq C_{t t} E^{1 / \kappa}|x-y|^{\lambda / \kappa},
$$

so by (34) $1-C_{t p} E^{1 / \kappa}\left(2 C_{t p} C_{0}|x-y|^{\lambda / \kappa}\right) \leq C_{t t} E^{1 / \kappa}|x-y|^{\lambda / \kappa}$;

hence $\quad|x-y| \geq \hat{C}(m, l, p) E^{-1 / \lambda}$.

We set $R_{2}=\frac{1}{2} \min \left\{\tilde{R}_{2}\left(C_{0} C_{t p}\right)^{-1}, \hat{C}(m, l, p) E^{-1 / \lambda}\right\}$.

Corollary 4.9. For each $x \in \Sigma$ and each $y \in \Sigma \cap \overline{\mathbb{B}}\left(x, R_{2}\right)$ the point $y$ is the only point in the intersection $\Sigma \cap\left(y+T_{x} \Sigma^{\perp}\right) \cap \overline{\mathbb{B}}\left(x, R_{2}\right)$. Therefore $(\Sigma-x) \cap \overline{\mathbb{B}}_{R}$ is a graph of the function

$$
\begin{aligned}
F_{x}: \mathcal{D}(x) & \rightarrow T_{x} \Sigma^{\perp} \cap \overline{\mathbb{B}}_{R_{2}} \quad \text { defined by } \\
F_{x}(w)+w & =(\Sigma-x) \cap\left(w+T_{x} \Sigma^{\perp}\right) \cap \overline{\mathbb{B}}_{R_{2}},
\end{aligned}
$$

where $\mathcal{D}(x)=\pi_{x}\left(\Sigma \cap \overline{\mathbb{B}}_{R_{2}}\right) \subseteq T_{x} \Sigma$. By Theorem 1 the function $F_{x}$ is of class $C^{1, \lambda / \kappa}$.

Fix a point $o \in \Sigma$. We define the parameterization

$$
\varphi: \mathcal{D}(o) \rightarrow \Sigma \cap \overline{\mathbb{B}}\left(o, R_{2}\right) \quad \text { by } \quad \varphi(x)=o+F_{o}(x)+x .
$$

Recall our convention, that when we write $T_{o} \Sigma$ we always mean the appropriate subspace of $\mathbb{R}^{n}$. For $x \in \mathcal{D}(o)$ we set

$$
L_{x}=\left(\left.\pi_{o}\right|_{T_{\varphi(x)} \Sigma}\right)^{-1}: T_{o} \Sigma \rightarrow T_{\varphi(x)} \Sigma
$$

and

$$
K_{x}=\left(\left.\pi_{o}^{\perp}\right|_{T_{\varphi(x)} \Sigma^{\perp}}\right)^{-1}: T_{o} \Sigma^{\perp} \rightarrow T_{\varphi(x)} \Sigma^{\perp} .
$$

Observe that these mappings are well defined since $R_{2}$ is not greater than $\tilde{R}_{2}$ defined in Remark 4.7, which ensures that $d_{\mathrm{Gr}}\left(T_{o} \Sigma, T_{\varphi(x)} \Sigma\right) \leq \iota$. Note also that for any unit vector $v \in T_{\varphi(x)} \Sigma$ we have $\left|\pi_{o}^{\perp} v\right|=\left|\pi_{o} v-\pi_{\varphi(x)} v\right| \leq \iota$; hence $\left|\pi_{o} v\right|=\left|v-\pi_{o}^{\perp} v\right| \geq 1-\iota$. This shows that the norms $\left\|L_{x}\right\|_{T_{o} \Sigma}$ and $\left\|K_{x}\right\|_{T_{o} \Sigma^{\perp}}$ are less or equal to $(1-\iota)^{-1}$.

Remark 4.10. Recall that $\iota<\frac{1}{2}$. For $x \in \mathcal{D}(o)$ and $h \in T_{o} \Sigma$ we have (cf. [13. Lemma 3.15])

$$
\begin{aligned}
& \qquad D F_{o}(x) h=L_{x} h-h=\pi_{o}^{\perp}\left(L_{x} h\right) \quad \text { and } \quad D \varphi(x) h=L_{x} h ; \\
& \text { hence }\left\|D F_{o}(x)\right\| \leq \frac{\iota}{1-\iota}<2 \iota<1 \quad \text { and } \quad\|D \varphi(x)\| \leq \frac{1}{1-\iota}<1+2 \iota<2 .
\end{aligned}
$$


Remark 4.11. For all $x \in \mathcal{D}(o)$ we have $\|D \varphi(x)\|<2$ and in consequence $|\varphi(x)-\varphi(o)|<2|x-o|$. Hence $T_{o} \Sigma \cap \overline{\mathbb{B}}_{\frac{1}{2} R_{2}} \subseteq \mathcal{D}(o)$.

Lemma 4.12. Let $C_{\rho \varepsilon}$ be the constant from Proposition 1.16 , For any $x, y \in \mathcal{D}(o)$ we have

$$
\begin{aligned}
\|D \varphi(x)-D \varphi(y)\| & \leq 4 d_{\mathrm{Gr}}\left(T_{\varphi(x)} \Sigma, T_{\varphi(y)} \Sigma\right) \\
\text { and } \quad d_{\mathrm{Gr}}\left(T_{\varphi(x)} \Sigma, T_{\varphi(y)} \Sigma\right) & \leq C_{\rho \varepsilon}\|D \varphi(x)-D \varphi(y)\| .
\end{aligned}
$$

Proof. We want to estimate

$$
\|D \varphi(x)-D \varphi(y)\|=\left\|D F_{o}(x)-D F_{o}(y)\right\|=\left\|L_{x}-L_{y}\right\| .
$$

Let $h \in \mathbb{S}$ and set $u=L_{x}(h)$ and $v=L_{y}(h)$. Note that $u-v \in T_{o} \Sigma^{\perp}$ and that $|v| \leq 2$ and also $d_{\mathrm{Gr}}\left(T_{\varphi(x)} \Sigma, T_{o} \Sigma\right) \leq \frac{1}{2}$, so we can write

$$
\begin{aligned}
\left|L_{x}(h)-L_{y}(h)\right| & \left.=|u-v| \leq 2 \mid \pi_{\varphi(x)}^{\perp}(u-v)\right)|=2| \pi_{\varphi(x)}^{\perp}(v) \mid \\
& \leq 2|v| d_{\operatorname{Gr}}\left(T_{\varphi(x)} \Sigma, T_{\varphi(y)} \Sigma\right) \leq 4 d_{\operatorname{Gr}}\left(T_{\varphi(x)} \Sigma, T_{\varphi(y)} \Sigma\right) .
\end{aligned}
$$

To prove the second part of Lemma 4.12 we will use Proposition 1.16. Let $\left(e_{1}, \ldots, e_{m}\right)$ be some orthonormal basis of $T_{o} \Sigma$. For each $i=1, \ldots, m$ set $u_{i}=$ $D \varphi(x) e_{i}$ and $v_{i}=D \varphi(y) e_{i}$. Then $\left(u_{1}, \ldots, u_{m}\right)$ is a basis of $T_{\varphi(x)} \Sigma$ and $\left(v_{1}, \ldots, v_{m}\right)$ is a basis of $T_{\varphi(y)} \Sigma$. By Remark 4.10 for $i, j=1, \ldots, m$ and $i \neq j$ we have

$$
1 \leq\left|e_{i}+D F_{o}(x) e_{i}\right|=\left|u_{i}\right|=\left|D \varphi(x) e_{i}\right| \leq \frac{1}{1-\iota}<1+2 \iota
$$

and $\left|\left\langle u_{i}, u_{j}\right\rangle\right|=\left|\left\langle D F_{o}(x) e_{i}+e_{i}, D F_{o}(x) e_{j}+e_{j}\right\rangle\right| \leq 4 \iota^{2}+2 \iota+2 \iota<6 \iota$.

These estimates show that $\left(u_{1}, \ldots, u_{m}\right)$ is a $\rho \varepsilon$-basis of $T_{\varphi(x)} \Sigma$ with $\rho=1$ and $\varepsilon=6 \iota$. Moreover

$$
\left|u_{i}-v_{i}\right|=\left|D \varphi(x) e_{i}-D \varphi(y) e_{i}\right| \leq\|D \varphi(x)-D \varphi(y)\| .
$$

Since $6 \iota=\frac{6}{100} \boldsymbol{\epsilon}_{\rho \varepsilon} \leq \boldsymbol{\epsilon}_{\rho \varepsilon}$ we can use Proposition 1.16 to obtain

$$
d_{\mathrm{Gr}}\left(T_{\varphi(x)} \Sigma, T_{\varphi(y)} \Sigma\right) \leq C_{\rho \varepsilon}\|D \varphi(x)-D \varphi(y)\| .
$$

Proof of Theorem 4.1. Combining Lemma 4.6 with Lemma 4.12 we get

$$
\left\|D F_{o}(x)-D F_{o}(y)\right\|=\|D \varphi(x)-D \varphi(y)\| \leq 4 C_{t t} E^{1 / \kappa}|x-y|^{\lambda / \kappa}
$$

for all $x, y \in \mathcal{D}(o)=\pi_{o}\left(\Sigma \cap \overline{\mathbb{B}}_{R_{2}}\right) \subseteq T_{o} \Sigma$. Since $\pi_{o}$ is continuous and $\Sigma \cap \overline{\mathbb{B}}_{R_{2}}$ is compact, the function $F_{o}: \mathcal{D}(o) \rightarrow T_{o} \Sigma^{\perp}$ can be extended to a function $F_{o}$ : $T_{o} \Sigma \rightarrow T_{o} \Sigma^{\perp}$ without increasing Hölder norm of its derivative and in such a way that $\left\{y+F_{o}(y): y \in T_{o} \Sigma \backslash \mathcal{D}(o)\right\} \cap \overline{\mathbb{B}}_{R_{2}}=\emptyset$. Hence we may set

$$
R_{\lambda \kappa}=R_{2}=C(m, l, p) E^{-\frac{1}{\lambda}} \quad \text { and } \quad C_{\lambda \kappa}=4 C_{t t} E^{\frac{1}{\kappa}} .
$$

\section{Optimal Hölder Regularity}

In the previous paragraph we showed that $\Sigma$ is a closed manifold of class $C^{1, \lambda / \kappa}$ but $\lambda / \kappa$ was not an optimal exponent. Now we shall prove that for any $o \in \Sigma$ the map $F_{o}$ is of class $C^{1, \alpha}$, where $\alpha=1-\frac{m l}{p}$. For this purpose we employ a technique developed by Strzelecki, Szumańska and von der Mosel in [24].

The key to the proof of Theorem 3 is Lemma 5.1. It says that the oscillation of $D \varphi$ on ball of radius $r$ can be bounded above by the oscillation of $D \varphi$ on a ball of radius $r / N$, where $N$ is some big number, plus a term of order $r^{\alpha}$. If we choose 
$N$ big enough, then, upon iteration, the first term disappears and the sum of the second terms is still of order $r^{\alpha}$.

To prove Lemma 5.1 we choose two points $x, y \in \mathcal{D}(o)$ and we set $r=|x-y|$. From Lemma 4.12 we know that the oscillation of $D \varphi$ is comparable with the oscillation of $T_{\varphi(\cdot)} \Sigma$. We choose points $x_{0}, \ldots, x_{m}$ and $y_{0}, \ldots, y_{m}$ near $x$ and $y$ respectively, such that $\left\{x_{i}-x_{0}\right\}_{i=1}^{m}$ and $\left\{y_{i}-y_{0}\right\}_{i=1}^{m}$ form a roughly (up to an error of order $\frac{1}{k}$, where $k$ is some big number) orthogonal bases of $T_{o} \Sigma$. Moreover $\left|x_{i}-x_{0}\right| \approx r / N$ and $\left|y_{i}-y_{0}\right| \approx r / N$. In the scale we are working in, we always have $\|D \varphi\| \leq 1+\iota$, so $\left\{\varphi\left(x_{i}\right)-\varphi\left(x_{0}\right)\right\}_{i=1}^{m}$ and $\left\{\varphi\left(y_{i}\right)-\varphi\left(y_{0}\right)\right\}_{i=1}^{m}$ are also roughly (up to an error of order $\left.\frac{1}{k}+\iota\right)$ orthogonal and span some $m$-dimensional secant spaces $X$ and $Y$ respectively. If we choose the points $y_{0}, \ldots, y_{m}$ appropriately, then the "angle" $d_{\mathrm{Gr}}(X, Y)$ can be estimated by $r^{\alpha}$. The error we make when we pass from $d_{\mathrm{Gr}}\left(T_{\varphi(x}, T_{\varphi(y)}\right)$ to $d_{\mathrm{Gr}}(X, Y)$ is comparable with the oscillation of $D \varphi$ on balls of radius $r / N$.

To choose "good" points $y_{0}, \ldots, y_{m}$ we first define the set of "bad parameters" $\mathfrak{B}\left(x_{0}, \ldots, x_{l-2}\right)$, i.e. such $z \in \mathcal{D}(o)$ that the integrand

$$
\mathcal{K}_{l, \varphi}\left(x_{0}, \ldots, x_{l-2}, z\right)=\sup _{p_{l}, \ldots, p_{m+1} \in \Sigma} \mathcal{K}\left(\varphi\left(x_{0}\right), \ldots, \varphi\left(x_{l-2}\right), z, p_{l}, \ldots, p_{m+1}\right)
$$

is big. From finiteness of $\mathcal{E}_{p}^{l}(\Sigma)$, we derive the conclusion that the measure of $\mathfrak{B}\left(x_{0}, \ldots, x_{l-2}\right)$ has to be smaller than the measure of a ball of radius $r /(k N)$; hence close to any $\tilde{p} \in \mathcal{D}(o)$ there exists $p \in \mathcal{D}(o)$ which does not belong to $\mathfrak{B}\left(x_{0}, \ldots, x_{l-2}\right)$. From the fact that $\mathcal{K}_{l, \varphi}\left(x_{0}, \ldots, x_{l-2}, y\right)$ is small, we derive an estimate $\operatorname{dist}\left(\varphi(y), \varphi\left(x_{0}\right)+X\right) \lesssim r^{1+\alpha}$, which in turn gives $d_{\mathrm{Gr}}(X, Y) \lesssim r^{\alpha}$.

In the sequel of this section we always assume that $\Sigma$ satisfies the hypotheses of Theorem 4.1, $o \in \Sigma$ is fixed, $\varphi$ is given by (38) and $l$ is a fixed number from the set $\{1,2, \ldots, m+2\}$.

5.1. Bootstrapping the Hölder exponent. Let $S \subseteq \mathcal{D}(o)$ be any set and $r \leq$ $\frac{1}{2} R_{\lambda \kappa}$. We define the oscillation of $D \varphi$ on $S$ as follows:

$$
\Phi(r, S)=\sup \{\|D \varphi(x)-D \varphi(y)\|: x, y \in S,|x-y| \leq r\} .
$$

For $x, y \in T_{o} \Sigma$ we set

$$
\mathbb{D}_{r}=T_{o} \Sigma \cap \mathbb{B}_{r}, \quad \mathbb{D}(x, r)=x+\mathbb{D}_{r} \quad \text { and } \quad \mathfrak{D}(x, y)=\mathbb{D}_{|x-y|}+\frac{x+y}{2} \subseteq T_{o} \Sigma,
$$

and we define

$$
M_{p}^{l}(a, \rho)=\left(\mathcal{E}_{p}^{l}(\varphi(\overline{\mathbb{D}}(a, \rho)))\right)^{\frac{1}{p}} \quad \text { and } \quad E_{p}^{l}(x, y)=\mathcal{E}_{p}^{l}(\varphi(\overline{\mathfrak{D}}(x, y))) .
$$

Note that if we set $|J \varphi(x)|=\sqrt{\operatorname{det}\left((D \varphi(x))^{t} D \varphi(x)\right)}$ and

$$
\mathcal{K}_{l, \varphi}\left(x_{0}, \ldots, x_{l-1}\right)=\sup _{p_{l}, \ldots, p_{m+1} \in \Sigma} \mathcal{K}\left(\varphi\left(x_{0}\right), \ldots, \varphi\left(x_{l-1}\right), p_{l}, \ldots, p_{m+1}\right),
$$

then $E_{p}^{l}(x, y)=\int_{[\overline{\mathfrak{D}}(x, y)]^{l}} \mathcal{K}_{l, \varphi}\left(x_{0}, \ldots, x_{l-1}\right)^{p}\left|J \varphi\left(x_{0}\right)\right| \cdots\left|J \varphi\left(x_{l-1}\right)\right| d x_{0} \cdots d x_{l-1}$.

Lemma 5.1. For all $k \geq k_{0}=100 / \epsilon_{\rho \varepsilon}$ and $N \geq N_{0}=8$ there exist constants $C_{1}=C_{1}(m)$ and $C_{2}=C_{2}(m, l, p, k, N)$ such that for all $x, y \in \mathbb{D}_{\frac{1}{6} R_{\lambda \kappa}}$

$$
\|D \varphi(x)-D \varphi(y)\| \leq C_{1} \Phi\left(\frac{2|x-y|}{N}, \overline{\mathfrak{D}}(x, y)\right)+C_{2} E_{p}^{l}(x, y)^{\frac{1}{p}}|x-y|^{\alpha} .
$$

Using this lemma we can prove Theorem 3 . 
Proof of Theorem 3. Fix some $a \in \overline{\mathbb{D}}_{\frac{1}{12}} R_{\lambda \kappa}$ and a radius $R \in\left(0, \frac{1}{36} R_{\lambda \kappa}\right]$. Taking the supremum on both sides of (40) over all $x, y \in \overline{\mathbb{D}}(a, R)$ satisfying $|x-y| \leq r \leq R$ we obtain the estimate

$$
\Phi(r, \overline{\mathbb{D}}(a, R)) \leq C_{1} \Phi\left(\frac{2 r}{N}, \overline{\mathbb{D}}(a, R+r)\right)+C_{2} M_{p}^{l}(a, R+r) r^{\alpha} .
$$

Choose any $j \in \mathbb{N}$. Iterating the above inequality $j$ times we get

$$
\Phi(r, \overline{\mathbb{D}}(a, R)) \leq C_{1}^{j} \Phi\left(2^{j} N^{-j} r, \overline{\mathbb{D}}\left(R+r_{j}\right)\right)+C_{2} M_{p}\left(a, R+r_{j}\right) r^{\alpha} \sum_{l=0}^{j-1}\left(\frac{C_{1}}{N^{\alpha}}\right)^{l},
$$

where $r_{j}=r \sum_{l=0}^{j-1} 2^{l} N^{-l} \leq 2 r$. Recall that we know a priori that $\varphi$ is $C^{1, \lambda / \kappa_{-}}$ smooth, so we can estimate the first term on the right-hand side by

$$
\Phi\left(2^{j} N^{-j} r, \overline{\mathbb{D}}\left(a, R+r_{j}\right)\right) \leq C_{\lambda \kappa} 2^{j \lambda / \kappa} N^{-j \lambda / \kappa} r^{\lambda / \kappa},
$$

which gives

$$
\Phi(r, \overline{\mathbb{D}}(a, R)) \leq C_{\lambda \kappa} 2^{j \lambda / \kappa}\left(C_{1} N^{-\lambda / \kappa}\right)^{j} r^{\lambda / \kappa}+C_{2} M_{p}^{l}(a, 3 R) r^{\alpha} \sum_{l=0}^{j-1}\left(C_{1} N^{-\alpha}\right)^{l}
$$

for each $j \in \mathbb{N}$. To ensure that the first term disappears and that the second term converges when $j \rightarrow \infty$ we need to know the following:

$$
C_{1} 2^{\lambda / \kappa} N^{-\lambda / \kappa}<1 \text { and } C_{1} N^{-\alpha}<1 \text {. }
$$

Since $C_{1}=C_{1}(m)$, we can find $N=N(m, l, p) \geq N_{0}$ for which condition (41) is satisfied. Passing with $j$ to the limit $j \rightarrow \infty$ we obtain the bound

$$
\Phi(r, \overline{\mathbb{D}}(a, R)) \leq C_{2} M_{p}^{l}(a, 3 R) \sum_{l=0}^{\infty}\left(C_{1} N^{-\alpha}\right)^{l} r^{\alpha}=C(m, l, p) M_{p}^{l}(a, 3 R) r^{\alpha} .
$$

Hence, for any $x, y \in \overline{\mathbb{D}}_{\frac{1}{36} R_{\lambda \kappa}}$, taking $a=\frac{x+y}{2}$ and $r=R=|x-y|$ we get

$$
\|D \varphi(x)-D \varphi(y)\| \leq C(m, l, p) M_{p}^{l}\left(\frac{x+y}{2}, 3|x-y|\right)|x-y|^{\alpha} .
$$

Proof of Lemma 5.1. Let us fix $x, y \in \mathbb{D}_{\frac{1}{6} R_{\lambda \kappa}}$. Since $|x-y|<\frac{1}{3} R_{\lambda \kappa}$ and $\frac{|x+y|}{2}<$ $\frac{1}{6} R_{\lambda \kappa}$, we have $\mathfrak{D}(x, y) \subseteq \mathbb{D}_{\frac{1}{2} R_{\lambda \kappa}}$. Let $x_{0}, \ldots, x_{l-2} \in \mathfrak{D}(x, y)$. If $l \geq 2$ we define the set of bad parameters as

$$
\mathfrak{B}\left(x_{0}, \ldots, x_{l-2}\right)=\left\{z \in \overline{\mathfrak{D}}(x, y): \mathcal{K}_{l, \varphi}\left(x_{0}, \ldots, x_{l-2}, z\right)^{p}>\frac{(k N)^{m}}{|x-y|^{m l} \omega_{m}^{l}} E_{p}^{l}(x, y)\right\}
$$

and in case $l=1$ we set

$$
\mathfrak{B}=\left\{z \in \overline{\mathfrak{D}}(x, y): \mathcal{K}_{l, \varphi}(z)^{p}>\frac{(k N)^{m}}{|x-y|^{m} \omega_{m}} E_{p}^{1}(x, y)\right\} .
$$

Recalling (39) and using the fact that $|J \varphi| \geq 1$ we can estimate the measure of $\mathfrak{B}\left(x_{0}, \ldots, x_{l-2}\right)$ as follows:

$$
\begin{aligned}
E_{p}^{l}(x, y) & \geq \int_{[\mathfrak{D}(x, y)]^{l-1}} \int_{\mathfrak{B}\left(x_{0}, \ldots, x_{l-2}\right)} \mathcal{K}_{l, \varphi}\left(x_{0}, \ldots, x_{l-2}, z\right) d z d x_{0} \cdots d x_{l-2} \\
& >\omega_{m}^{l-1}|x-y|^{m(l-1)} \mathcal{H}^{m}\left(\mathfrak{B}\left(x_{0}, \ldots, x_{l-2}\right)\right) \frac{(k N)^{m}}{|x-y|^{m l} \omega_{m}^{l}} E_{p}^{l}(x, y) \\
& \Longleftrightarrow \mathcal{H}^{m}\left(\mathfrak{B}\left(x_{0}, \ldots, x_{l-2}\right)\right)<\omega_{m}\left(\frac{|x-y|}{k N}\right)^{m} .
\end{aligned}
$$


Fix an orthonormal basis $\left(e_{1}, \ldots, e_{m}\right)$ of $T_{o} \Sigma$. For $i=1, \ldots, m$ we set

$$
x_{0}=x, \quad x_{i}=x_{0}+\frac{|x-y|}{N} e_{i}, \quad \tilde{y}_{0}=y \quad \text { and } \quad \tilde{y}_{i}=\tilde{y}_{0}+\frac{|x-y|}{N} e_{i} .
$$

Estimate (42) shows that we can find

$$
y_{0}, \ldots, y_{m} \in \overline{\mathfrak{D}}(x, y) \backslash \mathfrak{B}\left(x_{0}, \ldots, x_{l-2}\right), \quad \text { such that } \quad\left|y_{i}-\tilde{y}_{i}\right| \leq \frac{|x-y|}{k N}
$$

for each $i=0, \ldots, m$. We set

$$
X=\operatorname{span}\left\{\varphi\left(x_{i}\right)-\varphi\left(x_{0}\right)\right\}_{i=1}^{m} \quad \text { and } \quad Y=\operatorname{span}\left\{\varphi\left(y_{i}\right)-\varphi\left(y_{0}\right)\right\}_{i=1}^{m} .
$$

Using Lemma 4.12 we obtain

$$
\begin{aligned}
& \|D \varphi(x)-D \varphi(y)\| \leq\left\|D \varphi(x)-D \varphi\left(x_{0}\right)\right\|+\left\|D \varphi\left(x_{0}\right)-D \varphi\left(y_{0}\right)\right\|+\left\|D \varphi\left(y_{0}\right)-D \varphi(y)\right\| \\
& \leq 4 d_{\mathrm{Gr}}\left(T_{\varphi\left(x_{0}\right)} \Sigma, T_{\varphi\left(y_{0}\right)} \Sigma\right)+\Phi\left(\frac{|x-y|}{k N}, \overline{\mathfrak{D}}(x, y)\right) \\
& \leq \Phi\left(\frac{|x-y|}{k N}, \overline{\mathfrak{D}}(x, y)\right)+4 d_{\mathrm{Gr}}\left(T_{\varphi\left(x_{0}\right)} \Sigma, X\right)+4 d_{\mathrm{Gr}}(X, Y)+4 d_{\mathrm{Gr}}\left(Y, T_{\varphi\left(y_{0}\right)} \Sigma\right) .
\end{aligned}
$$

For each $i=1, \ldots, m$, from the fundamental theorem of calculus we have

$$
\begin{aligned}
v_{i} & =\varphi\left(x_{i}\right)-\varphi\left(x_{0}\right)=\int_{0}^{1} \frac{d}{d t}\left(\varphi\left(x_{0}+t\left(x_{i}-x_{0}\right)\right)\right) d t \\
& =\int_{0}^{1}\left(D \varphi\left(x_{0}+t\left(x_{i}-x_{0}\right)\right)-D \varphi\left(x_{0}\right)\right)\left(x_{i}-x_{0}\right) d t+D \varphi\left(x_{0}\right)\left(x_{i}-x_{0}\right) \\
& =\sigma_{i}+w_{i} .
\end{aligned}
$$

Observe that $w_{1}, \ldots, w_{m}$ form a basis of $T_{\varphi\left(x_{0}\right)} \Sigma$ and $v_{1}, \ldots, v_{m}$ span $X$. Using the above estimate we see that

$$
\left|v_{i}-w_{i}\right|=\left|\sigma_{i}\right| \leq \Phi\left(\left|x_{i}-x_{0}\right|, \mathfrak{D}(x, y)\right)\left|x_{i}-x_{0}\right|=\Phi\left(\frac{|x-y|}{N}, \mathfrak{D}(x, y)\right) \frac{|x-y|}{N} .
$$

Let $a_{i}=x_{i}-x_{0}=\frac{|x-y|}{N} e_{i}$ and $b_{i}=F_{o}\left(x_{i}\right)-F_{o}\left(x_{0}\right)$. Then $v_{i}=a_{i}+b_{i}$. From Remark 4.10 we know that $\left|b_{i}\right| \leq 2 \iota\left|a_{i}\right|=\frac{|x-y|}{50 N} \boldsymbol{\epsilon}_{\rho \varepsilon}$; hence

$$
\begin{aligned}
\frac{|x-y|^{2}}{N^{2}}\left(\delta_{i}^{j}-\frac{\boldsymbol{\epsilon}_{\rho \varepsilon}}{25}-\frac{\boldsymbol{\epsilon}_{\rho \varepsilon}^{2}}{50^{2}}\right) & \leq\left|\left\langle v_{i}, v_{j}\right\rangle\right|=\left|\left\langle a_{i}+b_{i}, a_{j}+b_{j}\right\rangle\right| \\
& \leq \frac{|x-y|^{2}}{N^{2}}\left(\delta_{i}^{j}+\frac{\boldsymbol{\epsilon}_{\rho \varepsilon}}{25}+\frac{\boldsymbol{\epsilon}_{\rho \varepsilon}^{2}}{50^{2}}\right) .
\end{aligned}
$$

Applying Proposition 1.16 we come to

$$
d_{\mathrm{Gr}}\left(T_{\varphi\left(x_{0}\right)} \Sigma, X\right) \leq C_{\rho \varepsilon} \Phi\left(\frac{|x-y|}{N}, \mathfrak{D}(x, y)\right) .
$$

We estimate $d_{\mathrm{Gr}}\left(T_{\varphi\left(y_{0}\right)} \Sigma, Y\right)$ in a similar way. For $i=1, \ldots, m$ we define $\bar{v}_{i}, \bar{w}_{i}$, $\bar{a}_{i}$ and $\bar{b}_{i}$ as follows:

$$
\begin{gathered}
\bar{a}_{i}=y_{i}-y_{0}, \quad \bar{b}_{i}=F_{o}\left(y_{i}\right)-F_{o}\left(y_{0}\right), \\
\bar{v}_{i}=\varphi\left(y_{i}\right)-\varphi\left(y_{0}\right)=\bar{a}_{i}+\bar{b}_{i} \quad \text { and } \quad \bar{w}_{i}=D \varphi\left(y_{0}\right)\left(y_{i}-y_{0}\right),
\end{gathered}
$$

so that $Y=\operatorname{span}\left\{\bar{v}_{1}, \ldots, \bar{v}_{m}\right\}$ and $T_{\varphi\left(y_{0}\right)} \Sigma=\operatorname{span}\left\{\bar{w}_{1}, \ldots, \bar{w}_{m}\right\}$. Again, using the fundamental theorem of calculus, we get

$$
\left|\bar{v}_{i}-\bar{w}_{i}\right| \leq \Phi\left(\left|y_{i}-y_{0}\right|, \mathfrak{D}(x, y)\right)\left|y_{i}-y_{0}\right| \leq 2 \Phi\left(\frac{2|x-y|}{N}, \mathfrak{D}(x, y)\right) \frac{|x-y|}{N} .
$$


Recall that $k \geq 100 / \boldsymbol{\epsilon}_{\rho \varepsilon}$. It is easy to verify that

$$
\frac{|x-y|^{2}}{N^{2}}\left(\delta_{i}^{j}-\frac{8}{k}\right) \leq\left|\left\langle\bar{a}_{i}, \bar{a}_{j}\right\rangle\right| \leq \frac{|x-y|^{2}}{N^{2}}\left(\delta_{i}^{j}+\frac{8}{k}\right),
$$

which implies that $\left|\bar{b}_{i}\right| \leq 2 \iota\left|\bar{a}_{i}\right| \leq \frac{|x-y|}{25 N} \boldsymbol{\epsilon}_{\rho \varepsilon}$. Therefore

$$
\frac{|x-y|^{2}}{N^{2}}\left(\delta_{i}^{j}-\boldsymbol{\epsilon}_{\rho \varepsilon}\right) \leq\left|\left\langle\bar{v}_{i}, \bar{v}_{j}\right\rangle\right|=\left|\left\langle\bar{a}_{i}+\bar{b}_{i}, \bar{a}_{j}+\bar{b}_{j}\right\rangle\right| \leq \frac{|x-y|^{2}}{N^{2}}\left(\delta_{i}^{j}+\boldsymbol{\epsilon}_{\rho \varepsilon}\right)
$$

and we can apply Proposition 1.16 once more obtaining

$$
d_{\mathrm{Gr}}\left(T_{\varphi\left(y_{0}\right)} \Sigma, Y\right) \leq 2 C_{\rho \varepsilon} \Phi\left(\frac{2|x-y|}{N}, \mathfrak{D}(x, y)\right) .
$$

Combining estimates (45), (44) and (43) and using Lemma 4.12 we get

$$
\|D \varphi(x)-D \varphi(y)\| \leq C_{1}(m) \Phi\left(\frac{2|x-y|}{k N}, \overline{\mathfrak{D}}(x, y)\right)+4 d_{\mathrm{Gr}}(X, Y) .
$$

Hence, we only need to estimate $d_{\mathrm{Gr}}(X, Y)$.

Observe that for each $z \in \mathfrak{D}(x, y) \backslash \mathfrak{B}\left(x_{0}, \ldots, x_{l-2}\right)$ we have

$$
\mathcal{K}_{l, \varphi}\left(x_{0}, \ldots, x_{l-2}, z\right) \leq \frac{(k N)^{m / p}}{\omega_{m}^{l / p}|x-y|^{m l / p}} E_{p}^{l}(x, y)^{1 / p} .
$$

Using the following basic formula for the measure of a simplex $\triangle\left(p_{0}, \ldots, p_{m+1}\right) \subset$ $\mathbb{R}^{n}$

$$
\mathcal{H}^{m+1}\left(\triangle\left(p_{0}, \ldots, p_{m+1}\right)\right)=\frac{1}{m+1} \mathcal{H}^{m}\left(\triangle\left(p_{0}, \ldots, p_{m}\right)\right) \operatorname{dist}\left(p_{m+1}, \operatorname{aff}\left\{p_{0}, \ldots, p_{m}\right\}\right)
$$

and using the definition of $\mathcal{K}_{l, \varphi}$ we can write

$$
\begin{gathered}
\mathcal{K}_{l, \varphi}\left(x_{0}, \ldots, x_{l-2}, z\right) \geq \mathcal{K}\left(\varphi\left(x_{0}\right), \ldots, \varphi\left(x_{m}\right), \varphi(z)\right) \\
=\frac{\mathcal{H}^{m}\left(\triangle\left(\varphi\left(x_{0}\right), \ldots, \varphi\left(x_{m}\right)\right) \operatorname{dist}\left(\varphi(z), \varphi\left(x_{0}\right)+X\right)\right)}{(m+1) \operatorname{diam}\left(\triangle\left(\varphi\left(x_{0}\right), \ldots, \varphi\left(x_{m}\right), \varphi(z)\right)\right)^{m+2}} \\
\geq \frac{\mathcal{H}^{m}\left(\triangle\left(x_{0}, \ldots, x_{m}\right)\right) \operatorname{dist}\left(\varphi(z), \varphi\left(x_{0}\right)+X\right)}{(m+1)(2|x-y|)^{m+2}}=\frac{\operatorname{dist}\left(\varphi(z), \varphi\left(x_{0}\right)+X\right)}{(m+1) ! N^{m} 2^{m+2}|x-y|^{2}} .
\end{gathered}
$$

Putting (47) and (48) together we get

$$
\operatorname{dist}\left(\varphi(z), \varphi\left(x_{0}\right)+X\right) \leq C(m, l, p, k, N) E_{p}^{l}(x, y)^{1 / p}|x-y|^{1-\frac{m l}{p}} \frac{|x-y|}{N} .
$$

We have shown already that $\bar{v}_{1}, \ldots, \bar{v}_{m}$ forms a $\rho \varepsilon$-basis of $Y$ with $\rho=\frac{|x-y|}{N}$ and $\varepsilon=\boldsymbol{\epsilon}_{\rho \varepsilon}$. Moreover, since $y_{i} \notin \mathfrak{B}\left(x_{0}, \ldots, x_{l-2}\right)$, we have

$$
\begin{aligned}
\operatorname{dist}\left(\bar{v}_{i}, X\right)=\left|\pi_{X}^{\perp} \bar{v}_{i}\right| & \leq \operatorname{dist}\left(\varphi\left(y_{i}\right), \varphi\left(x_{0}\right)+X\right)+\operatorname{dist}\left(\varphi\left(y_{0}\right), \varphi\left(x_{0}\right)+X\right) \\
& \leq 2 C(m, l, p, k, N) E_{p}^{l}(x, y)^{1 / p}|x-y|^{1-\frac{m l}{p}} \frac{|x-y|}{N}
\end{aligned}
$$

Thence, by Proposition 1.16 the following holds:

$$
d_{\mathrm{Gr}}(X, Y) \leq \tilde{C}(m, l, p, k, N) E_{p}^{l}(x, y)^{1 / p}|x-y|^{1-\frac{m l}{p}} .
$$

Together with (46) this gives (40) and Lemma 5.1 is proven. 


\section{ACKNOWLEDGEMEnTS}

Much of the material presented in this article was contained in the author's doctoral thesis written at the University of Warsaw under the direction of Pawel Strzelecki. The author wishes to thank Professor Strzelecki for all his helpful advice and encouragement.

The author also wishes to thank the anonymous reviewer who very carefully read the first version of this paper and had many constructive suggestions.

\section{REFERENCES}

[1] Simon Blatt and Sławomir Kolasiński, Sharp boundedness and regularizing effects of the integral Menger curvature for submanifolds, Adv. Math. 230 (2012), no. 3, 839-852, DOI 10.1016/j.aim.2012.03.007. MR2921162

[2] Simon Blatt, A note on integral Menger curvature for curves, Math. Nachr. 286 (2013), no. 2-3, 149-159, DOI 10.1002/mana.201100220. MR3021472

[3] Guy David, Carlos Kenig, and Tatiana Toro, Asymptotically optimally doubling measures and Reifenberg flat sets with vanishing constant, Comm. Pure Appl. Math. 54 (2001), no. 4, 385-449, DOI 10.1002/1097-0312(200104)54:4¡385::AID-CPA1¿3.0.CO;2-M. MR.1808649 (2002g:28007)

[4] Guy David and Stephen Semmes, Analysis of and on uniformly rectifiable sets, Mathematical Surveys and Monographs, vol. 38, American Mathematical Society, Providence, RI, 1993. MR 1251061 (94i:28003)

[5] Herbert Federer, Curvature measures, Trans. Amer. Math. Soc. 93 (1959), 418-491. MR0110078 (22 \#961)

[6] O. Gonzalez, J. H. Maddocks, F. Schuricht, and H. von der Mosel, Global curvature and self-contact of nonlinearly elastic curves and rods, Calc. Var. Partial Differential Equations 14 (2002), no. 1, 29-68, DOI 10.1007/s005260100089. MR 1883599 (2002m:74035)

[7] Oscar Gonzalez and John H. Maddocks, Global curvature, thickness, and the ideal shapes of knots, Proc. Natl. Acad. Sci. USA 96 (1999), no. 9, 4769-4773 (electronic), DOI 10.1073/pnas.96.9.4769. MR.1692638 (2000c:57008)

[8] Allen Hatcher, Algebraic topology, Cambridge University Press, Cambridge, 2002. MR 1867354 (2002k:55001)

[9] Morris W. Hirsch, Differential topology, Springer-Verlag, New York, 1976. Graduate Texts in Mathematics, No. 33. MR.0448362 (56 \#6669)

[10] Peter W. Jones, The traveling salesman problem and harmonic analysis, Publ. Mat. 35 (1991), no. 1, 259-267, DOI 10.5565/PUBLMAT_35191_12. Conference on Mathematical Analysis (El Escorial, 1989). MR1103619 (92c:42013)

[11] S. Kolasiński, P. Strzelecki, and H. von der Mosel, Compactness for the class of manifolds with equibounded curvature energy, in preparation.

[12] Sławomir Kolasiński, Paweł Strzelecki, and Heiko von der Mosel, Characterizing $W^{2, p}$ submanifolds by p-integrability of global curvatures, Geom. Funct. Anal. 23 (2013), no. 3, 937984, DOI 10.1007/s00039-013-0222-y. MR3061777

[13] Sławomir Kolasiński, Integral Menger curvature for sets of arbitrary dimension and codimension. PhD thesis, Institute of Mathematics, University of Warsaw, 2011, arXiv:1011.2008.

[14] Sławomir Kolasiński and Marta Szumańska, Minimal Hölder regularity implying finiteness of integral Menger curvature, Manuscripta Math. 141 (2013), no. 1-2, 125-147, DOI 10.1007/s00229-012-0565-y. MR3042684

[15] J. C. Léger, Menger curvature and rectifiability, Ann. of Math. (2) 149 (1999), no. 3, 831-869, DOI 10.2307/121074. MR1709304(2001c:49069)

[16] Gilad Lerman and J. Tyler Whitehouse, High-dimensional Menger-type curvatures. II. dseparation and a menagerie of curvatures, Constr. Approx. 30 (2009), no. 3, 325-360, DOI 10.1007/s00365-009-9073-z. MR2558685 (2011c:60041)

[17] Gilad Lerman and J. Tyler Whitehouse, High-dimensional Menger-type curvatures. Part I: Geometric multipoles and multiscale inequalities, Rev. Mat. Iberoam. 27 (2011), no. 2, 493555, DOI 10.4171/RMI/645. MR2848529(2012m:28006) 
[18] L. S. Pontryagin, Selected works. Vol. 3, Classics of Soviet Mathematics, Gordon \& Breach Science Publishers, New York, 1986. Algebraic and differential topology; Edited and with a preface by R. V. Gamkrelidze; Translated from the Russian by P. S. V. Naidu. MR.898008 (90a:01107)

[19] E. R. Reifenberg, Solution of the Plateau Problem for $m$-dimensional surfaces of varying topological type, Acta Math. 104 (1960), 1-92. MR0114145(22 \#4972)

[20] Sebastian Scholtes, For which positive $p$ is the integral Menger curvature $\mathcal{M}_{p}$ finite for all simple polygons?, 2012, arXiv:1202.0504.

[21] Sebastian Scholtes, Tangency properties of sets with finite geometric curvature energies, Fund. Math. 218 (2012), no. 2, 165-191, DOI 10.4064/fm218-2-4. MR2957689

[22] Leon Simon, Reifenberg's topological disc theorem, 1996. Mathematisches Institut Universität Tübingen. Preprints AB Analysis.

[23] Paweł Strzelecki, Marta Szumańska, and Heiko von der Mosel, A geometric curvature double integral of Menger type for space curves, Ann. Acad. Sci. Fenn. Math. 34 (2009), no. 1, 195-214. MR2489022 (2009m:28016)

[24] Pawel Strzelecki, Marta Szumańska, and Heiko von der Mosel, Regularizing and self-avoidance effects of integral Menger curvature, Ann. Sc. Norm. Super. Pisa Cl. Sci. (5) 9 (2010), no. 1, 145-187. MR2668877 (2011j:28009)

[25] Paweł Strzelecki and Heiko von der Mosel, On rectifiable curves with $L^{p}$-bounds on global curvature: self-avoidance, regularity, and minimizing knots, Math. Z. 257 (2007), no. 1, 107130, DOI 10.1007/s00209-007-0117-4. MR2318572(2008e:49019)

[26] Paweł Strzelecki and Heiko von der Mosel, Integral Menger curvature for surfaces, Adv. Math. 226 (2011), no. 3, 2233-2304, DOI 10.1016/j.aim.2010.09.016. MR2739778

[27] Paweł Strzelecki and Heiko von der Mosel, Tangent-point repulsive potentials for a class of non-smooth $m$-dimensional sets in $\mathbb{R}^{n}$. Part I: Smoothing and self-avoidance effects, J. Geom. Anal. 23 (2013), no. 3, 1085-1139, DOI 10.1007/s12220-011-9275-z. MR3078345

Institute of Mathematics, University of WARsaw, Banacha 2, 02-097 Warsaw, Poland Current address: Max Planck Institute for Gravitational Physics, Albert Einstein Institute, Am Mühlenberg 1, D-14476 Golm, Germany

E-mail address: s.kolasinski@mimuw.edu.pl

$U R L:$ http://www.mimuw.edu.pl/ skola 